\title{
max \\ On Complex Numbers in Higher Dimensions
}

\author{
Wolf-Dieter Richter
}

check for updates

Citation: Richter, W.-D. On Complex Numbers in Higher Dimensions. Axioms 2022, 11, 22. https://doi.org/ 10.3390/axioms11010022

Academic Editor: Andriy Bandura

Received: 3 November 2021

Accepted: 28 December 2021

Published: 7 January 2022

Publisher's Note: MDPI stays neutral with regard to jurisdictional claims in published maps and institutional affiliations.

Copyright: (C) 2022 by the author Licensee MDPI, Basel, Switzerland. This article is an open access article distributed under the terms and conditions of the Creative Commons Attribution (CC BY) license (https:// creativecommons.org/licenses/by/ $4.0 /)$.
Institute of Mathematics, University of Rostock, 18051 Rostock, Germany; wolf-dieter.richter@uni-rostock.de; Tel.: +49-381-498-6551

\begin{abstract}
The geometric approach to generalized complex and three-dimensional hyper-complex numbers and more general algebraic structures being based upon a general vector space structure and a geometric multiplication rule which was only recently developed is continued here in dimension four and above. To this end, the notions of geometric vector product and geometric exponential function are extended to arbitrary finite dimensions and some usual algebraic rules known from usual complex numbers are replaced with new ones. An application for the construction of directional probability distributions is presented.
\end{abstract}

Keywords: geometric vector product; geometric vector power; geometric exponential function; vector division; spherical geometric coordinate product; spherical geometric coordinate vector ratio; multi-complex algebraic structure; multi-complex numbers; Euler-type representation; directional probability law

MSC: 11H99; 11L03; 60E05

\section{Introduction}

Quaternions, going back to Hamilton 1843, and the work of Gauss being unpublished until 1900, are four-dimensional hyper-complex numbers $x=x_{1}+x_{2} i+x_{3} j+x_{4} k$ where $x_{1}, \ldots, x_{4}$ are real numbers and $i, j, k$ are the fundamental quaternion units satisfying the Hamilton rules

$$
i^{2}=j^{2}=k^{2}=-1
$$

and

$$
i j k=-1,
$$

for example see [1,2]. Four alternative four-dimensional types of hyper-complex numbers are introduced in [3]. The rules of the so-called circular and hyperbolic fourcomplex numbers defined there are

$$
i^{2}=j^{2}=-1, k^{2}=1, i j=j i=-k, i k=k i=j, j k=k j=i
$$

and

$$
i^{2}=j^{2}=k^{2}=1, i j=j i=k, i k=k i=j, j k=k j=i,
$$

respectively, and so-called planar and polar fourcomplex numbers satisfy similar rules. In each of the mentioned cases, the product of the two hyper-complex numbers $x$ and $x^{\prime}=x_{1}^{\prime}+x_{2}^{\prime} i+x_{3}^{\prime} j+x_{4}^{\prime} k$ can be obtained by applying the mentioned rules together with the usual algebraic rules, known from the real and usual complex numbers, to the product

$$
x x^{\prime}=\left(x_{1}+x_{2} i+x_{3} j+x_{4} k\right)\left(x_{1}^{\prime}+x_{2}^{\prime} i+x_{3}^{\prime} j+x_{4}^{\prime} k\right) .
$$

On the one hand, out of habit, one could consider this approach to be natural, on the other hand, one could also ask for a content-related motivation for it. 
Another approach to multidimensional hyperbolic complex numbers is presented in [4]. In [5], a complex vector is dealt with as a sum of a vector and a so-called bivector. Moreover, numerous applications in electromagnetic theory and quantum mechanics are given there.

Bicomplex numbers studied in [6-8] are applied to quantum mechanics and quantum fields in [9-11] and can be represented as

$$
w=z_{1}+z_{2} i_{2}
$$

where $z_{1}$ and $z_{2}$ are elements of independent complex planes possessing imaginary units $i_{1}$ and $i_{2}$ which satisfy

$$
i_{1}^{2}=i_{2}^{2}=-1
$$

and

$$
i_{1} i_{2}=j, i_{1} j=-i_{2}, i_{2} j=-i_{1} .
$$

Here, $j$ satisfies

$$
j^{2}=1
$$

and is called a hyperbolic unit. For another application of bicomplex numbers we refer to the work in [12].

There are always several ways of generalizing or transferring a mathematical statement that is valid in one-dimensional to higher dimensions. If one specializes in (5), for example, $x_{3}=x_{4}=0$, the result is very reminiscent of the usual complex plane. The decisive question in a generalization process is which of the properties valid in the one-dimensional case should also apply in the higher-dimensional case.

In the language of the usual complex numbers, the four-complex numbers under consideration here also formally satisfy the rules (1) and

$$
i j=i k=j k=-1
$$

as well as a further specific algebraic rule which, inter alia, does not allow the implementation of all the usual conclusions from (1) and (6) as for complex numbers. The main aim of a study which was started in [13-15] and is continued here is to exemplarily show that there are infinitely many systems of generalized complex numbers of every dimension, and how to construct some of them in a largely standardized way. While complex numbers of dimension three were dealt with in [14], the present work deals with complex numbers in dimensions four and above. The general structure of a vector space and a multiplication rule being different from (5) and being specifically chosen in each case form the basic elements of our constructions. Based upon a respective multiplication rule, a generalized exponential function is introduced and with its help the Euler formula is generalized. The question of which multiplication rule is appropriate in which application area depends to a considerable extent on our detailed knowledge of the given situation or even requires an experimental answer, which, however, is outside the scope of the present work.

If $x=x_{1}+x_{2} i$ and $x^{\prime}=x_{1}^{\prime}+x_{2}^{\prime} i$ denote usual complex numbers then the product used in [13] in the case $p=2$ is

$$
x \odot x^{\prime}=\left(\begin{array}{l}
x_{1} x_{1}^{\prime}-x_{2} x_{2}^{\prime} \\
x_{1} x_{2}^{\prime}+x_{1}^{\prime} x_{2}
\end{array}\right),
$$

and if $x=x_{1}+x_{2} i+x_{3} j$ and $x^{\prime}=x_{1}^{\prime}+x_{2}^{\prime} i+x_{3}^{\prime} j$ are three-complex numbers then the corresponding multiplication rule defined in [14] is

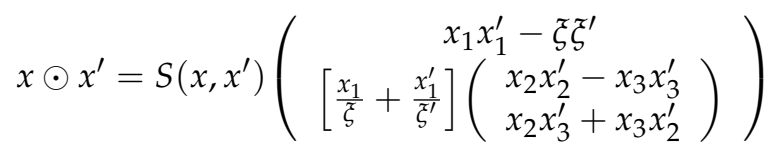


with

$$
S\left(x, x^{\prime}\right)=I_{[0, \pi]}\left(\varphi(x)+\varphi\left(x^{\prime}\right)\right)-I_{(\pi, 2 \pi)}\left(\varphi(x)+\varphi\left(x^{\prime}\right)\right)
$$

and

$$
\varphi(x)=\arctan \frac{\xi}{x_{1}}, \xi=\sqrt{x_{2}^{2}+x_{3}^{2}}, \quad \varphi\left(x^{\prime}\right)=\arctan \frac{\xi^{\prime}}{x_{1}^{\prime}}, \xi^{\prime}=\sqrt{x_{2}^{\prime 2}+x_{3}^{\prime 2}} .
$$

Note that, for every $p>0, p$-generalizations of the multiplication rules (7) and (8) are also introduced in $[13,14]$, respectively. The technical background of the derivation of these rules consists in using spherical and $l_{p}$-spherical coordinates according to $[16,17]$, respectively. While Euclidean unit circles and unit spheres play a certain role in the visualization of complex numbers of various dimensions, their role in the visualization of $p$-generalized complex numbers is taken over by $l_{p}$-circles and $l_{p}$-spheres. In this framework, multiplication is a superposition of movements along longitudes and latitudes of $l_{p}$-spheres and scalings along rays through the origin. Its inversion, therefore, allows a geometrical explanation of the corresponding division rules.

With regard to the general structural properties, we emphasize that the multiplication rule of quaternions is not commutative, but the rule for the four-complex numbers considered here is.

Multidimensional constructions of complex structures follow the same line as in dimensions two up to four. The definition of the finite-dimensional spherical geometric vector product which is the heart of the present construction of multi-complex structures is addressed in Section 7 of the present work and is possibly even more of interest for vector analysis in general than just for the present straightforward constructions.

Finally, we note that, as discussed in Remark 4 in [14], the notion of a division algebra does not apply to a situation where common multiplication of complex numbers is replaced with a new one as in the present case. That is why the classical classification of real division algebras presented in [18] does not apply to the present framework but instead could serve as an orientation for some future work in the present new field of work.

The rest of the paper is structured as follows. We introduce an abstract four-complex algebraic structure in Section 2 and subsequently give a proof of the existence of realizations of it by presenting suitable examples. To be more specific, we define four-complex numbers in Section 3, present their probabilistic application in Section 4 and a geometric look at them in Section 5, study classes of $l_{p}^{4}$-complex numbers, $p>0$, in Section 6 and the finitedimensional spherical geometric vector product in Section 7. Multi-complex numbers are introduced in Section 8. The paper is completed by a short discussion in Section 9 and Appendix A providing additional material dealing with some consequences from filling a gap of mathematical rigor in the widespread literature on complex numbers.

\section{The Abstract Four-Complex Structure}

Let $V$ be a four-dimensional vector space endowed with an addition $\oplus: V \times V \rightarrow V$, a scalar multiplication $\bullet: V \times \mathbb{R} \rightarrow V$ and an additional vector multiplication $\circledast: V \times V \rightarrow V$. Assume that addition and vector multiplication are commutative and that addition is also associative. Let further $\mathfrak{o}$ and $\mathfrak{e}$ denote neutral elements with respect to addition and vector multiplication, respectively, and assume that there exist elements $i, j$ and $\mathfrak{k}$ of $V$ satisfying

$$
\mathfrak{i} \circledast \mathfrak{i}=\mathfrak{j} \circledast \mathfrak{j}=\mathfrak{k} \circledast \mathfrak{k}=\mathfrak{i} \circledast \mathfrak{j}=\mathfrak{i} \circledast \mathfrak{k}=\mathfrak{j} \circledast \mathfrak{k}=-\mathfrak{e} .
$$

Then $\mathbb{C}_{4}=(V, \oplus, \bullet, \circledast, \mathfrak{o}, \mathfrak{e}, \mathfrak{i}, \mathfrak{j}, \mathfrak{k})$ is called a four-complex structure.

Remark 1. Following standard notation in complex number theory, $i, j$ and $\mathfrak{k}$ might be called the imaginary units of the four-complex structure $\mathbb{C}_{4}$. 


\section{Four-Complex Numbers}

We are going now to prove that there exists a realization of the four-complex structure introduced in the previous section. To this end, let $\mathbb{R}^{4}$ be the four-dimensional Euclidean space, $\mathfrak{x}=x_{1} \mathfrak{e} \boxplus x_{2} \mathfrak{i} \boxplus x_{3} \mathfrak{j} \boxplus x_{4} \mathfrak{k}$ the standard orthonormal basis representation of $\mathfrak{x} \in \mathbb{R}^{4}$ with

$$
\mathfrak{e}=(1,0,0,0)^{T}, \mathfrak{i}=(0,1,0,0)^{T}, \mathfrak{j}=(0,0,1,0)^{T}, \mathfrak{k}=(0,0,0,1)
$$

and $\mathfrak{x}_{1} \boxplus \mathfrak{x}_{2}=\left(x_{1,1}+x_{1,2}, x_{2,1}+x_{2,2}, x_{3,1}+x_{3,2}, x_{4,1}+x_{4,2}\right)$ where $\mathfrak{x}_{l}=\left(x_{1, l}, x_{2, l}, x_{3, l}, x_{4, l}\right)^{T}$, $l=1,2$ the common (component-wise) vector addition. Then $\left(\mathbb{R}^{4}, \boxplus\right.$ ) is an Abelian group with the neutral element $\mathcal{O}=(0,0,0,0)^{T}$, and the additive inverse element of $\mathfrak{x}=\left(x_{1}, x_{2}, x_{3}, x_{4}\right)^{T}$ is $-\mathfrak{x}=\left(-x_{1},-x_{2},-x_{3},-x_{4}\right)^{T}$.

The following definition makes use of the notation

$$
\xi_{1, l}=\sqrt{x_{2, l}^{2}+x_{3, l}^{2}+x_{4, l}^{2}}, \xi_{2, l}=\sqrt{x_{3, l}^{2}+x_{4, l}^{2}}, l=1,2,
$$

and, for $m=1,2$,

$$
S_{m}\left(\mathfrak{x}_{1}, \mathfrak{x}_{2}\right)=I_{[0, \pi]}\left(\arctan \frac{\xi_{m, 1}}{x_{m, 1}}+\arctan \frac{\xi_{m, 2}}{x_{m, 2}}\right)-I_{(\pi, 2 \pi)}\left(\arctan \frac{\xi_{m, 1}}{x_{m, 1}}+\arctan \frac{\xi_{m, 2}}{x_{m, 2}}\right)
$$

where $I_{A}($.$) denotes the indicator function of the set A$.

Definition 1. Unless for

$$
\left(x_{3, l}, x_{4, l}\right)=(0,0) \text { or }\left(x_{2, l}, x_{3, l}, x_{4, l}\right)=(0,0,0) \text { for at least one value of } l \in\{1,2\}
$$

the geometric vector product of $\mathfrak{x}_{1}$ and $\mathfrak{x}_{2}$ is defined by

$\mathfrak{x}_{1} \odot \mathfrak{x}_{2}=S_{1}\left(\mathfrak{x}_{1}, \mathfrak{x}_{2}\right)\left(\begin{array}{c}x_{1,1} x_{1,2}-\xi_{1,1} \xi_{1,2} \\ x_{2,1} x_{2,2}-\xi_{2,1} \xi_{2,2} \\ S_{2}\left(\mathfrak{x}_{1}, \mathfrak{x}_{2}\right)\left[\frac{x_{1,1}}{\xi_{1,1}}+\frac{x_{1,2}}{\xi_{1,2}}\right]\left(\begin{array}{c}x_{3,1} x_{3,2}-x_{4,1} x_{4,2} \\ x_{3,1} x_{4,2}+x_{4,1} x_{3,2}\end{array}\right)\end{array}\right)$.

Moreover, we put

$$
\left(x_{1}, x_{2}, x_{3}, x_{4}\right)^{T} \odot(t, 0,0,0)^{T}=|t|\left(x_{1}, x_{2}, x_{3}, x_{4}\right)^{T}, t \in \mathbb{R}
$$

and

$$
\left(x_{1}, x_{2}, x_{3}, x_{4}\right)^{T} \odot(u, v, 0,0)^{T}=\left(x_{1} u-\left|x_{2}\right| v, x_{1} v+\left|x_{2}\right| u, 0,0\right)^{T}, t \in \mathbb{R} .
$$

Because of its symmetric structure, this product is commutative.

The specialization of the vector product from Section $2, \circledast=\odot$, applies to the rest of the present section. Let $\|$.$\| denote the Euclidean norm in \mathbb{R}^{4}$.

Remark 2. If $\left\|\mathfrak{x}_{2}\right\|=1$ then $\left\|\mathfrak{x}_{1} \odot \mathfrak{x}_{2}\right\|=\left\|\mathfrak{x}_{1}\right\|$. That is, the set of all maps $\mathfrak{x} \rightarrow \mathfrak{x} \odot \mathfrak{x}_{2}, \mathfrak{x} \in \mathbb{R}^{3}$ with $\left\|\mathfrak{x}_{2}\right\|=1$ is the Lie group on the sphere $\left\{\mathfrak{x}:\|\mathfrak{x}\|=\left\|\mathfrak{x}_{1}\right\|\right\}$.

Definition 2. The $q^{\prime}$ th geometric power of vector $\mathfrak{x}$ and its complex exponential are defined as $\mathfrak{x}^{0}=\mathfrak{e}, \mathfrak{x}^{q}=\mathfrak{x}^{q-1} \odot \mathfrak{x}, q=1,2, \ldots$ and $e_{\odot}^{\mathfrak{x}}=\sum_{q=0}^{\infty} \frac{\mathfrak{x}^{q}}{q !}$, respectively.

Example 1. If $\mathfrak{x}=x_{2} \mathfrak{i}+x_{3} \mathfrak{j}+x_{4} \mathfrak{k}$ then

$$
\mathfrak{x}^{2 q}=(-1)^{q}\left(x_{2}^{2}+x_{3}^{2}+x_{4}^{2}\right)^{q} \mathfrak{e} \text { and } \mathfrak{x}^{2 q+1}=(-1)^{q}\left(x_{2}^{2}+x_{3}^{2}+x_{4}^{2}\right)^{q} \mathfrak{x}
$$


for $q=0,1,2, \ldots$

Example 2. Further particular multiplication results are

$$
\mathfrak{i} \odot \mathfrak{i}=\mathfrak{j} \odot \mathfrak{j}=\mathfrak{k} \odot \mathfrak{k}=\mathfrak{i} \odot \mathfrak{j}=\mathfrak{i} \odot \mathfrak{k}=\mathfrak{j} \odot \mathfrak{k}=-\mathfrak{e} .
$$

Remark 3. Let $\lambda$ be a positive real number, then

$$
\mathfrak{x}_{1} \odot\left(\lambda \mathfrak{x}_{2}\right)=\lambda \mathfrak{x}_{1} \odot \mathfrak{x}_{2}
$$

which means that the geometric vector product is positively homogeneous.

Theorem 1. The following Euler type formulas are true:

$$
\begin{aligned}
e_{\odot}^{\tau \mathfrak{h}} & =\cos \tau \mathfrak{e}+\sin \tau \mathfrak{h}, \mathfrak{h} \in\{\mathfrak{i}, \mathfrak{j}, \mathfrak{k}\}, \\
e_{\odot}^{x_{1} \mathfrak{i}+x_{2} \mathfrak{j}+x_{3} \mathfrak{k}} & =\left(\cos \sqrt{x_{1}^{2}+x_{2}^{2}+x_{3}^{2}}\right) \mathfrak{e}+\left(\sin \sqrt{x_{1}^{2}+x_{2}^{2}+x_{3}^{2}}\right) \frac{x_{1} \mathfrak{i}+x_{2} \mathfrak{j}+x_{3} \mathfrak{k}}{\sqrt{x_{1}^{2}+x_{2}^{2}+x_{3}^{2}}}, \\
e_{\odot}^{x_{1} \mathfrak{i}} \odot e_{\odot}^{x_{2} \mathfrak{j}} & =\cos \left(x_{1}+x_{2}\right) \mathfrak{e}+\sin \left(x_{1}+x_{2}\right) \mathfrak{j}, \\
e_{\odot}^{x_{1} \mathfrak{i}} \odot e_{\odot}^{x_{3} \mathfrak{k}} & =\cos \left(x_{1}+x_{3}\right) \mathfrak{e}+\sin \left(x_{1}+x_{3}\right) \mathfrak{k}, \\
e_{\odot}^{x_{2} \mathfrak{j}} \odot e_{\odot}^{x_{3} \mathfrak{k}} & =\cos \left(x_{2}+x_{3}\right) \mathfrak{e}-\sin \left(x_{2}+x_{3}\right) \mathfrak{i} .
\end{aligned}
$$

Proof. The proof of this theorem immediately follows that of Theorem 1 in [14] and is therefore omitted here.

One of the obvious conclusions from this theorem is that

$$
e_{\odot}^{x_{1} \mathfrak{i}+x_{2} \mathfrak{j}}=\left(\cos \sqrt{x_{1}^{2}+x_{2}^{2}}\right) \mathfrak{e}+\left(\sin \sqrt{x_{1}^{2}+x_{2}^{2}}\right) \frac{x_{1} \mathfrak{i}+x_{2} \mathfrak{j}}{\sqrt{x_{1}^{2}+x_{2}^{2}}} .
$$

Example 3. For $\mathfrak{x}=\left(x_{1}, x_{2}, x_{3}, x_{4}\right)^{T} \in \mathbb{R}^{4}, \mathfrak{x} \neq \mathfrak{o}$, let $\widetilde{\mathfrak{x}}=\frac{1}{\|\mathfrak{x}\|^{2}}\left(-x_{1}, x_{2},-x_{3}, x_{4}\right)^{T}$, then

$$
-(\mathfrak{x} \odot \widetilde{\mathfrak{x}})=\mathfrak{e}
$$

and

$$
-(\mathfrak{x} \odot \widetilde{\mathfrak{x}}) \neq \mathfrak{x} \odot(-\mathfrak{x}) .
$$

Definition 3. Following [14], we call $\mathfrak{\mathfrak { x }}$ the negative-inverse of $\mathfrak{x}$.

Example 4. The following particular result is true:

$$
\mathfrak{x}_{1} \odot \widetilde{\mathfrak{x}_{2}}=\frac{S_{1}\left(\mathfrak{x}_{1}, \widetilde{\mathfrak{x}_{2}}\right)}{\left\|\mathfrak{x}_{2}\right\|^{2}}\left(S_{2}\left(\mathfrak{x}_{1}, \widetilde{\mathfrak{x}_{2}}\right)\left[\frac{x_{1,1}}{\tilde{\xi}_{1,1}}-\frac{x_{1,2}}{\xi_{1,2}}\right]\left(\begin{array}{c}
-x_{1,1} x_{1,2}-\xi_{1,1} \xi_{1,2} \\
x_{2,1} x_{2,2}-\xi_{2,1} \xi_{2,2} \\
-x_{3,1} x_{3,2}-\xi_{4,1} \xi_{4,2} \\
\left.x_{2,1}+\frac{x_{2,2}}{\xi_{2,2}}\right]\left(\begin{array}{r}
\xi_{3,1} \xi_{4,2}-x_{4,1} x_{3,2}
\end{array}\right)
\end{array}\right)\right) .
$$

Definition 4. Unless condition (11) is met, the geometric vector ratio, $\mathfrak{x}_{1}$ devided by $\mathfrak{x}_{2}$, is defined by

$$
\mathfrak{x}_{1} \oslash \mathfrak{x}_{2}=\frac{S_{1}^{*}}{\left\|\mathfrak{x}_{2}\right\|^{2}}\left(\begin{array}{c}
x_{1,1} x_{1,2}+\xi_{1,1} \xi_{1,2} \\
x_{2,1} x_{2,2}+\xi_{2,1} \xi_{2,2} \\
S_{2}^{*}\left[\frac{x_{1,2}}{\xi_{1,2}}-\frac{x_{1,1}}{\xi_{1,1}}\right]
\end{array}\left(\begin{array}{c}
x_{3,1} x_{3,2}+x_{4,1} x_{4,2} \\
-x_{3,1} x_{4,2}+x_{4,1} x_{3,2}
\end{array}\right)\right)
$$


where

$$
S_{m}^{*}=I_{[0, \pi]}\left(\arctan \frac{\xi_{m, 1}}{x_{m, 1}}-\arctan \frac{\xi_{m, 2}}{x_{m, 2}}\right)-I_{[-\pi, 0)}\left(\arctan \frac{\xi_{m, 1}}{x_{m, 1}}-\arctan \frac{\xi_{m, 2}}{x_{m, 2}}\right), m=1,2 .
$$

Definition 5. $\left(\mathbb{R}^{4}, \boxplus, \bullet, \odot, \mathfrak{o}, \mathfrak{e}, \mathfrak{i}, \mathfrak{j}, \mathfrak{k}\right)$ is called the space of four-complex numbers.

The following proposition summarizes the results of this section.

Proposition 1. The space of four-complex numbers is a realization of the general four-complex algebraic structure $\mathbb{C}_{4}$.

Remark 4. Following Section 5 in [14], it is obvious how to construct many more realizations of the four-complex algebraic structure $\mathbb{C}_{4}$ by modifying its underlying vector space and the multiplication rule.

\section{Directional Probability Laws}

A generalized Euler type formula was used in [14] for the derivation of a class of directional probability laws on the Euclidean unit sphere in the three-dimensional Euclidean space $\mathbb{R}^{3}$. In this section, we construct probability distributions on the Euclidean unit sphere

$$
S_{3}=\left\{\left(x_{1}, x_{2}, x_{3}, x_{4}\right)^{T} \in \mathbb{R}^{4}: x_{1}^{2}+x_{2}^{2}+x_{3}^{2}+x_{4}^{2}=1\right\}
$$

in the four-dimensional Euclidean space $\mathbb{R}^{4}$. The construction method makes use of the second formula in Equation (17), but a modified method could also use one of the other formulas. Let $(\Omega, \mathfrak{A}, P)$ denote a probability space and $(X, Y, Z)^{T}: \Omega \rightarrow \mathbb{R}^{3}$ a random vector defined on it. The random vector

$$
\kappa=e_{\odot}^{X i+Y j+Z k}
$$

takes its values on $S_{3}$ and allows each of the following representations

$$
\begin{gathered}
\kappa=\kappa_{1} \mathfrak{e}+\kappa_{2} \mathfrak{i}+\kappa_{3} \mathfrak{j}+\kappa_{4} \mathfrak{k} \text { where } \kappa_{1}^{2}+\kappa_{2}^{2}+\kappa_{3}^{2}+\kappa_{4}^{2}=1, \\
\kappa=\cos R \mathfrak{e}+\sin R \frac{X \mathfrak{i}+Y \mathfrak{j}+Z \mathfrak{k}}{R}
\end{gathered}
$$

and

$$
\kappa=\cos \Phi_{1} \mathfrak{e}+\sin \Phi_{1}\left[\cos \Phi_{2} \mathfrak{i}+\sin \Phi_{2}\left\langle\cos \Phi_{3} \mathfrak{j}+\sin \Phi_{3} \mathfrak{k}\right\rangle\right]
$$

where $R=\sqrt{X^{2}+Y^{2}+Z^{2}}$ and the random angles $\Phi_{1}$ and $\Phi_{2}$ take values in the interval $[0, \pi]$, and $\Phi_{3}$ in $[0,2 \pi)$. The Equation (22) is due to the second Euler-type formula in (17) and (23) corresponds to the spherical coordinate transformation when the radius variable is equal to one. The random vector

$$
U=\left(\frac{X}{R}, \frac{Y}{R}, \frac{Z}{R}\right)^{T}
$$

can be considered as the central projection of $(X, Y, Z)^{T}$ onto the unit sphere

$$
S_{2}=\left\{\left(x_{1}, x_{2}, x_{3}\right)^{T} \in \mathbb{R}^{3}: x_{1}^{2}+x_{2}^{2}+x_{3}^{2}=1\right\}
$$

in $\mathbb{R}^{3}$. If $(X, Y, Z)^{T}$ follows a spherical distribution law then $U$ is uniformly distributed with respect to the Euclidean surface content measure on $S_{2}$. The role of the random angular variable $\Phi_{1}: \Omega \rightarrow[0, \pi)$ in Equation (23) is taken over by the radius variable $R$ in (22),

$$
\Phi_{1}=\left\{\begin{array}{lll}
R & \text { if } & 0 \leq R<\pi \\
R-l \pi & \text { if } & l \pi \leq R<(l+1) \pi, l=1,2, \ldots
\end{array}\right.
$$


In other words,

$$
\begin{aligned}
\Phi_{1} & =R(\bmod \pi) \\
& =\sum_{l=0}^{\infty}\left(\sqrt{X^{2}+Y^{2}+Z^{2}}-l \pi\right) I_{[l \pi,(l+1) \pi)}\left(\sqrt{X^{2}+Y^{2}+Z^{2}}\right) .
\end{aligned}
$$

In case $(X, Y, Z)^{T}$ has a three-dimensional normal distribution $N_{3}(\mu, \Sigma)$ with expectation $\mu$ and covariance matrix $\Sigma$, the distribution of $U$ is called projected normal or angular Gaussian or offset normal distribution, and that of $R_{w}=R(\bmod \pi)$ is a wrapped noncentral Chi-square distribution.

\section{Geometric View}

The aim of this section is to approach the four-complex numbers from a geometrical point if view. In this context, it should be remembered that geometrical considerations also form a basis of a classification of hyper-complex numbers. Let $M_{4}=[0, \infty) \times[0, \pi]^{\times 2} \times$ $[0,2 \pi)$. A well-known way to define a spherical coordinate transformation $\operatorname{Pol}_{(4)}: M_{4} \rightarrow$ $R^{4}$ is to put

$$
\operatorname{Pol}_{(4)}\left(r, \varphi_{1}, \varphi_{2}, \varphi_{3}\right)=r\left(\begin{array}{l}
\cos \varphi_{1} \\
\sin \varphi_{1} \cos \varphi_{2} \\
\sin \varphi_{1} \sin \varphi_{2} \cos \varphi_{3} \\
\sin \varphi_{1} \sin \varphi_{2} \sin \varphi_{3}
\end{array}\right)=\left(\begin{array}{l}
x_{1} \\
x_{2} \\
x_{3} \\
x_{4}
\end{array}\right)=\mathfrak{x}
$$

Even though there holds $\operatorname{Pol}_{(4)}\left(1,0, \varphi_{2}, \varphi_{3}\right)=\mathfrak{e}$ for all $\varphi_{2}, \varphi_{3}$, this transformation is a.e. invertible with the inverse transformation $\mathrm{Pol}_{(4)}^{-1}\left(x_{1}, x_{2}, x_{3}, x_{4}\right)$ allowing the representations

$$
\begin{aligned}
& r=\sqrt{x_{1}^{2}+x_{2}^{2}+x_{3}^{2}+x_{4}^{2}}, \\
& \cos \varphi_{1}=\frac{x_{1}}{r}, \quad \sin \varphi_{1}=\frac{\sqrt{x_{2}^{2}+x_{3}^{2}+x_{4}^{2}}}{r}, \varphi_{1}=\arctan \frac{\sqrt{x_{2}^{2}+x_{3}^{2}+x_{4}^{2}}}{x_{1}}, \\
& \cos \varphi_{2}=\frac{x_{2}}{\sqrt{x_{2}^{2}+x_{3}^{2}+x_{4}^{2}}}, \sin \varphi_{2}=\frac{\sqrt{x_{3}^{2}+x_{4}^{2}}}{\sqrt{x_{2}^{2}+x_{3}^{2}+x_{4}^{2}}}, \varphi_{2}=\arctan \frac{\sqrt{x_{3}^{2}+x_{4}^{2}}}{x_{2}}, \\
& \cos \varphi_{3}=\frac{x_{3}}{\sqrt{x_{3}^{2}+x_{4}^{2}}}, \quad \sin \varphi_{3}=\frac{x_{4}}{\sqrt{x_{3}^{2}+x_{4}^{2}}}, \quad \varphi_{3}=\arctan \frac{x_{4}}{x_{3}} \text {. }
\end{aligned}
$$

Example 5. The transformations $\mathrm{Pol}_{(4)}$ and $\mathrm{Pol}_{(4)}^{-1}$ give the following particular results:

and

$$
\begin{gathered}
\operatorname{Pol}_{(4)}\left(t, \frac{\pi}{2}, 0,0\right)=t \cdot \mathfrak{i}, \quad \operatorname{Pol}_{(4)}^{-1}(\mathfrak{i})=\left(1, \frac{\pi}{2}, 0,0\right), \\
\operatorname{Pol}_{(4)}\left(t, \frac{\pi}{2}, \frac{\pi}{2}, 0\right)=t \cdot \mathfrak{j}, \quad \operatorname{Pol}_{(4)}^{-1}(\mathfrak{j})=\left(1, \frac{\pi}{2}, \frac{\pi}{2}, 0\right)
\end{gathered}
$$

$$
\operatorname{Pol}_{(4)}\left(t, \frac{\pi}{2}, \frac{\pi}{2}, \frac{\pi}{2}\right)=t \cdot \mathfrak{k}, \quad \operatorname{Pol}_{(4)}^{-1}(\mathfrak{k})=\left(1, \frac{\pi}{2}, \frac{\pi}{2}, \frac{\pi}{2}\right) .
$$

Definition 6. The spherical coordinate product of the vectors $\operatorname{Pol}_{(4)}\left(r_{l}, \varphi_{1, l}, \varphi_{2, l}, \varphi_{3, l}\right), l=1,2$, is defined as

$$
\begin{aligned}
\operatorname{Pol}_{(4)}\left(r_{1}, \varphi_{1,1}, \varphi_{2,1}, \varphi_{3,1}\right) & * \operatorname{Pol}_{(4)}\left(r_{2}, \varphi_{1,2}, \varphi_{2,2}, \varphi_{3,2}\right) \\
= & \operatorname{Pol}_{(4)}\left(r_{1} r_{2}, \varphi_{1,1} \diamond \varphi_{1,2}, \varphi_{2,1} \diamond \varphi_{2,2}, \varphi_{3,1} \triangleright \varphi_{3,2}\right)
\end{aligned}
$$


where

$$
\psi \diamond \vartheta=(\psi+\vartheta) I_{[0, \pi]}(\psi+\vartheta)+(\psi+\vartheta-\pi) I_{(\pi, 2 \pi)}(\psi+\vartheta)
$$

and

$$
\psi \triangleright \vartheta=(\psi+\vartheta) I_{[0,2 \pi]}(\psi+\vartheta)+(\psi+\vartheta-2 \pi) I_{(2 \pi, 4 \pi)}(\psi+\vartheta) .
$$

Theorem 2. The spherical coordinate product of the vectors $\operatorname{Pol}_{(4)}\left(r_{l}, \varphi_{1, l}, \varphi_{2, l}, \varphi_{3, l}\right)=\left(x_{1, l}\right.$, $\left.x_{2, l}, x_{3, l}, x_{4, l}\right)^{T}=\mathfrak{x}_{l}, l=1,2$ according to Definition 6 coincides with their geometric vector product according to Definition 1.

Proof. According to Definition 6, the spherical coordinate product of the two vectors $\operatorname{Pol}_{(4)}\left(r_{1}, \varphi_{1,1}, \varphi_{2,1}, \varphi_{3,1}\right)$ and $\operatorname{Pol}_{(4)}\left(r_{2}, \varphi_{1,2}, \varphi_{2,2}, \varphi_{3,2}\right)$ equals

$$
\begin{aligned}
& \operatorname{Pol}_{(4)}\left(r_{1} r_{2}, \varphi_{1,1} \diamond \varphi_{1,2}, \varphi_{2,1} \diamond \varphi_{2,2}, \varphi_{3,1} \triangleright \varphi_{3,2}\right) \\
& =r_{1} r_{2}\left[I_{[0, \pi]}\left(\varphi_{1,1}+\varphi_{1,2}\right)\left(\begin{array}{l}
\cos \left(\varphi_{1,1}+\varphi_{1,2}\right) \\
\sin \left(\varphi_{1,1}+\varphi_{1,2}\right) \cos \left(\varphi_{2,1} \diamond \varphi_{2,2}\right) \\
\sin \left(\varphi_{1,1}+\varphi_{1,2}\right) \sin \left(\varphi_{2,1} \diamond \varphi_{2,2}\right) \cos \left(\varphi_{3,1} \triangleright \varphi_{3,2}\right) \\
\sin \left(\varphi_{1,1}+\varphi_{1,2}\right) \sin \left(\varphi_{2,1} \diamond \varphi_{2,2}\right) \sin \left(\varphi_{3,1} \triangleright \varphi_{3,2}\right)
\end{array}\right)\right. \\
& \left.\quad+I_{(\pi, 2 \pi)}\left(\varphi_{1,1}+\varphi_{1,2}\right)\left(\begin{array}{l}
\cos \left(\varphi_{1,1}+\varphi_{1,2}-\pi\right) \\
\sin \left(\varphi_{1,1}+\varphi_{1,2}-\pi\right) \cos \left(\varphi_{2,1} \diamond \varphi_{2,2}\right) \\
\sin \left(\varphi_{1,1}+\varphi_{1,2}-\pi\right) \sin \left(\varphi_{2,1} \diamond \varphi_{2,2}\right) \cos \left(\varphi_{3,1} \triangleright \varphi_{3,2}\right) \\
\sin \left(\varphi_{1,1}+\varphi_{1,2}-\pi\right) \sin \left(\varphi_{2,1} \diamond \varphi_{2,2}\right) \sin \left(\varphi_{3,1} \triangleright \varphi_{3,2}\right)
\end{array}\right)\right] .
\end{aligned}
$$

By (26) and (27), and with regard to the fact that the sine and cosine functions are $2 \pi$-periodic, we have that

$$
\begin{aligned}
& \operatorname{Pol}_{(4)}\left(r_{1} r_{2}, \varphi_{1,1} \diamond \varphi_{1,2}, \varphi_{2,1} \diamond \varphi_{2,2}, \varphi_{3,1} \triangleright \varphi_{3,2}\right) \\
& =r_{1} r_{2}\left[I_{1,1} I_{2,1}\left(\begin{array}{l}
\cos \left(\varphi_{1,1}+\varphi_{1,2}\right) \\
\sin \left(\varphi_{1,1}+\varphi_{1,2}\right) \cos \left(\varphi_{2,1}+\varphi_{2,2}\right) \\
\sin \left(\varphi_{1,1}+\varphi_{1,2}\right) \sin \left(\varphi_{2,1}+\varphi_{2,2}\right) \cos \left(\varphi_{3,1}+\varphi_{3,2}\right) \\
\sin \left(\varphi_{1,1}+\varphi_{1,2}\right) \sin \left(\varphi_{2,1}+\varphi_{2,2}\right) \sin \left(\varphi_{3,1}+\varphi_{3,2}\right)
\end{array}\right)\right. \\
& +I_{1,1} I_{2,2}\left(\begin{array}{l}
\cos \left(\varphi_{1,1}+\varphi_{1,2}\right) \\
\sin \left(\varphi_{1,1}+\varphi_{1,2}\right) \cos \left(\varphi_{2,1}+\varphi_{2,2}-\pi\right) \\
\sin \left(\varphi_{1,1}+\varphi_{1,2}\right) \sin \left(\varphi_{2,1}+\varphi_{2,2}-\pi\right) \cos \left(\varphi_{3,1}+\varphi_{3,2}\right) \\
\sin \left(\varphi_{1,1}+\varphi_{1,2}\right) \sin \left(\varphi_{2,1}+\varphi_{2,2}-\pi\right) \sin \left(\varphi_{3,1}+\varphi_{3,2}\right)
\end{array}\right) \\
& \left.\quad \begin{array}{l}
\cos \left(\varphi_{1,1}+\varphi_{1,2}-\pi\right) \\
\sin \left(\varphi_{1,1}+\varphi_{1,2}-\pi\right) \cos \left(\varphi_{2,1}+\varphi_{2,2}\right) \\
\sin \left(\varphi_{1,1}+\varphi_{1,2}-\pi\right) \sin \left(\varphi_{2,1}+\varphi_{2,2}\right) \cos \left(\varphi_{3,1}+\varphi_{3,2}\right) \\
\sin \left(\varphi_{1,1}+\varphi_{1,2}-\pi\right) \sin \left(\varphi_{2,1}+\varphi_{2,2}\right) \sin \left(\varphi_{3,1}+\varphi_{3,2}\right)
\end{array}\right) \\
& \left.\left.\begin{array}{l}
\cos \left(\varphi_{1,1}+\varphi_{1,2}-\pi\right) \\
\sin \left(\varphi_{1,1}+\varphi_{1,2}-\pi\right) \cos \left(\varphi_{2,1}+\varphi_{2,2}-\pi\right) \\
\sin \left(\varphi_{1,1}+\varphi_{1,2}-\pi\right) \sin \left(\varphi_{2,1}+\varphi_{2,2}-\pi\right) \cos \left(\varphi_{3,1}+\varphi_{3,2}\right) \\
\sin \left(\varphi_{1,1}+\varphi_{1,2}-\pi\right) \sin \left(\varphi_{2,1}+\varphi_{2,2}-\pi\right) \sin \left(\varphi_{3,1}+\varphi_{3,2}\right)
\end{array}\right)\right]
\end{aligned}
$$

with

$$
I_{l, 1}=I_{[0, \pi]}\left(\varphi_{l, 1}+\varphi_{l, 2}\right), I_{l, 2}=I_{(\pi, 2 \pi)}\left(\varphi_{l, 1}+\varphi_{l, 2}\right), l=1,2 .
$$

Because of the relationships $\cos (\psi-\pi)=-\cos \psi$ and $\sin (\psi-\pi)=-\sin \psi$ for $\psi \in(\pi, 2 \pi)$, it follows that 


$$
\begin{aligned}
& \operatorname{Pol}_{(4)}\left(r_{1} r_{2}, \varphi_{1,1} \diamond \varphi_{1,2}, \varphi_{2,1} \diamond \varphi_{2,2}, \varphi_{3,1} \triangleright \varphi_{3,2}\right)
\end{aligned}
$$

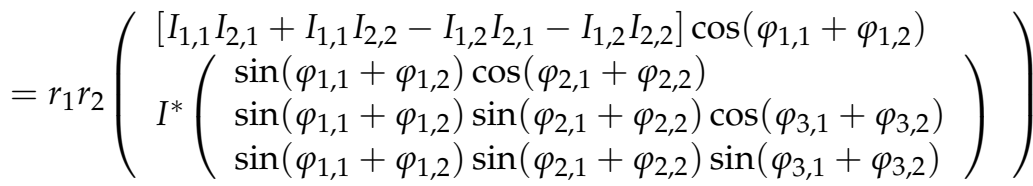

$$
\begin{aligned}
& =r_{1} r_{2}\left[I_{1,1}-I_{1,2}\right]\left(\begin{array}{c}
{\left[I_{2,1}+I_{2,2}\right] \cos \left(\varphi_{1,1}+\varphi_{1,2}\right)} \\
{\left[I_{2,1}-I_{2,2}\right]\left(\begin{array}{l}
\sin \left(\varphi_{1,1}+\varphi_{1,2}\right) \cos \left(\varphi_{2,1}+\varphi_{2,2}\right) \\
\sin \left(\varphi_{1,1}+\varphi_{1,2}\right) \sin \left(\varphi_{2,1}+\varphi_{2,2}\right) \cos \left(\varphi_{3,1}+\varphi_{3,2}\right) \\
\sin \left(\varphi_{1,1}+\varphi_{1,2}\right) \sin \left(\varphi_{2,1}+\varphi_{2,2}\right) \sin \left(\varphi_{3,1}+\varphi_{3,2}\right)
\end{array}\right)}
\end{array}\right) \\
& =r_{1} r_{2}\left[I_{1,1}-I_{1,2}\right]\left(\begin{array}{c}
c_{1,1} c_{1,2}-s_{1,1} s_{1,2} \\
c_{2,1} c_{2,2}-s_{2,1} s_{2,2} \\
{\left[I_{2,1}-I_{2,2}\right]\left(s_{1,1} c_{1,2}+c_{1,1} s_{1,2}\right)}
\end{array}\left(\begin{array}{c}
\left(s_{2,1} c_{2,2}+c_{2,1} s_{2,2}\right)\left(c_{3,1} c_{3,2}-s_{3,1} s_{3,2}\right) \\
\left(s_{2,1} c_{2,2}+c_{2,1} s_{2,2}\right)\left(s_{3,1} c_{3,2}+c_{3,1} s_{3,2}\right)
\end{array}\right)\right)
\end{aligned}
$$

where $I^{*}=\left[I_{1,1} I_{2,1}-I_{1,1} I_{2,2}-I_{1,2} I_{2,1}+I_{1,2} I_{2,2}\right]$ and $c_{v, l}=\cos \varphi_{v, l}, \quad s_{v, l}=\sin \varphi_{v, l}$ for $v=1,2,3$ and $l=1,2$. Inverse spherical coordinate transformation yields

$$
\begin{aligned}
\operatorname{Pol}_{(4)}\left(r_{1} r_{2}, \varphi_{1,1} \diamond \varphi_{1,2}, \varphi_{2,1} \diamond \varphi_{2,2}, \varphi_{3,1} \triangleright \varphi_{3,2}\right) \\
\quad=\left[I_{1,1}-I_{1,2}\right]\left(\begin{array}{c}
x_{1,1} x_{1,2}-\xi_{1,1} \xi_{1,2} \\
x_{2,1} x_{2,2}-\xi_{2,1} \xi_{2,2} \\
\frac{I_{2,1}-I_{2,2}}{\xi_{1,1} \xi_{1,2}}\left(\xi_{1,1} x_{1,2}+x_{1,1} \xi_{1,2}\right)\left(\begin{array}{c}
\left(\xi_{2,1} x_{2,2}+x_{2,1} \xi_{2,2}\right) \frac{x_{3,1} x_{3,2}-x_{4,1} x_{4,2}}{\xi_{2,1} \xi_{2,2}} \\
\left(\xi_{2,1} x_{2,2}+x_{2,1} \xi_{2,2}\right) \frac{x_{4,1} x_{3,2}+x_{3,1} x_{4,2}}{\xi_{2,1} \xi_{2,2}}
\end{array}\right)
\end{array}\right)
\end{aligned}
$$

proving that (25) and (12) describe the same multiplication.

Remark 5. (a) The spherical coordinate multiplication

$$
\mathfrak{x}=\operatorname{Pol}_{(4)}\left(r, \varphi_{1}, \varphi_{2}, \varphi_{3}\right) \rightarrow \operatorname{Pol}_{(4)}\left(r, \varphi_{1}, \varphi_{2}, \varphi_{3}\right) \odot \mathfrak{e}=\mathfrak{x}
$$

defines the identical map, the map

$$
\mathfrak{x} \rightarrow \mathfrak{x} \odot \mathfrak{i}=\operatorname{Pol}_{(4)}\left(r, \varphi_{1}+\frac{\pi}{2}, \varphi_{2}, \varphi_{3}\right)
$$

enlarges the angle between $\mathfrak{x}$ and $\mathfrak{e}$ by $\pi / 2$.

(b) Let $\Pi_{2,3,4}$ and $\Pi_{3,4}$ denote the results of orthogonally projecting $\mathfrak{x}$ into the subspaces of $\mathbb{R}^{4}$ spanned by $\mathfrak{i}, \mathfrak{j}$ and $\mathfrak{k}$ or by $\mathfrak{j}$ and $\mathfrak{k}$, respectively. The map

$$
\mathfrak{x} \rightarrow \mathfrak{x} \odot \mathfrak{j}=\operatorname{Pol}_{(4)}\left(r, \varphi_{1}+\frac{\pi}{2}, \varphi_{2}+\frac{\pi}{2}, \varphi_{3}\right)
$$

both enlarges the angle between $\mathfrak{x}$ and $\mathfrak{e}$ by $\pi / 2$ and that between $\Pi_{2,3,4}$ and $i$ also by $\pi / 2$. Moreover, the map

$$
\mathfrak{x} \rightarrow \mathfrak{x} \odot \mathfrak{k}=\operatorname{Pol}_{(4)}\left(r, \varphi_{1}+\frac{\pi}{2}, \varphi_{2}+\frac{\pi}{2}, \varphi_{3}+\frac{\pi}{2}\right)
$$

enlarges each of the angles between $\mathfrak{x}$ and $\mathfrak{e}, \Pi_{2,3,4}$ and $i$ as well as that between $\Pi_{3,4}$ and $j$ by $\pi / 2$.

Remark 6. The space of four-complex numbers may be alternatively represented as

$$
\left(\left\{\operatorname{Pol}_{(4)}\left(r, \varphi_{1}, \varphi_{2}, \varphi_{3}\right), \varphi_{l} \in[0, \pi), l=1,2, \varphi_{3} \in[0,2 \pi), r>0\right\}, \boxplus, \bullet, *, \mathfrak{o}, \mathfrak{e}, \mathfrak{i}, \mathfrak{j}, \mathfrak{k}\right)
$$


with elements $\mathfrak{e}, \mathfrak{i}, \mathfrak{j}, \mathfrak{k}$ satisfying the equations in (16) and (18). In this consideration, the radius parameter $r$ is multiplicative and the three angular parameters $\varphi_{1}, \varphi_{2}, \varphi_{3}$ are additive upon the spherical coordinate multiplication.

Remark 7. If $\mathfrak{x}=\operatorname{Pol}_{(4)}\left(r, \varphi_{1}, \varphi_{2}, \varphi_{3}\right)$ then $\widetilde{\mathfrak{x}}=\operatorname{Pol}_{(4)}\left(r, \pi-\varphi_{1}, \varphi_{2}, \pi-\varphi_{3}\right)$, thus (18), illustrating Example 3 from a geometric point of view.

Definition 7. The spherical coordinate vector ratio, vector $\operatorname{Pol}_{(4)}\left(r_{1}, \varphi_{1,1}, \varphi_{2,1}, \varphi_{3,1}\right)$ divided by the vector $\operatorname{Pol}_{(4)}\left(r_{2}, \varphi_{1,2}, \varphi_{2,2}, \varphi_{3,2}\right)$, is defined as

$$
\begin{aligned}
& \operatorname{Pol}_{(4)}\left(r_{1}, \varphi_{1,1}, \varphi_{2,1}, \varphi_{3,1}\right) \div \operatorname{Pol}_{(4)}\left(r_{2}, \varphi_{1,2}, \varphi_{2,2}, \varphi_{3,2}\right) \\
= & \operatorname{Pol}_{(4)}\left(\frac{r_{1}}{r_{2}}, \varphi_{1,1} \star \varphi_{1,2}, \varphi_{2,1} \star \varphi_{2,2}, \varphi_{3,1}-\varphi_{3,2}\right)
\end{aligned}
$$

where

$$
\varphi_{l} \star \vartheta_{l}=\left(\varphi_{l}-\vartheta_{l}\right) I_{[0, \pi]}\left(\varphi_{l}-\vartheta_{l}\right)+\left(\varphi_{l}-\vartheta_{l}+\pi\right) I_{[-\pi, 0)}\left(\varphi_{l}-\vartheta_{l}\right), l=1,2
$$

Theorem 3. The spherical coordinate ratio of the two vectors $\operatorname{Pol}_{(4)}\left(r_{l}, \varphi_{1, l}, \varphi_{2, l}, \varphi_{3, l}\right)=$ $\left(x_{1, l}, x_{2, l}, x_{3, l}, x_{4, l}\right)=\mathfrak{x}_{l}, l=1,2$ according to Definition 7 coincides with their geometric vector ratio according to Definition 4.

Proof. The proof of this theorem is quite similar to that of Theorem 2 and therefore be omitted here.

\section{Classes of Four-Complex Numbers}

Matrix, polynomial, and variable basis representations of three-complex numbers have been considered in [14] and can be proved for four-complex numbers, too, showing that there exist many more realizations of the four-complex structure introduced in Section 2. Such representations allow numerous modifications which may be of particular interest in specific applied situations and are left to the reader. The purpose of this section is to go one step further in discussing the existence of fundamentally different types of realizations of the general four-complex structure. We recall that according to Section 3, multiplying two four-complex numbers who's radius variables attain the value one means a movement on the Euclidean unit sphere. That's why we speak of this case as of the Euclidean one, while the present section deals with non-Euclidean cases whenever $p \neq 2$.

We start this section with an analytical consideration. Let $p>0$. For $\mathfrak{x}=\left(x_{1}, \ldots, x_{4}\right)^{T}$, from now on let

$$
\xi_{1}=\left(\left|x_{2}\right|^{p}+\left|x_{3}\right|^{p}+\left|x_{4}\right|^{p}\right)^{1 / p}, \xi_{2}=\left(\left|x_{3}\right|^{p}+\left|x_{4}\right|^{p}\right)^{1 / p}
$$

and

$$
\Gamma_{l}=\left|\left(\begin{array}{c}
\xi_{l, 1} x_{l, 2}+x_{l, 1} \xi_{l, 2} \\
x_{l, 1} x_{l, 2}-\xi_{l, 1} \xi_{l, 2}
\end{array}\right)\right|_{p}, l=1,2, \Gamma_{3}=\left|\left(\begin{array}{c}
x_{4,1} x_{3,2}+x_{3,1} x_{4,2} \\
x_{3,1} x_{3,2}-x_{4,1} x_{4,2}
\end{array}\right)\right|_{p} .
$$

Definition 8. Unless condition (11) is met, the geometric vector p-product $\mathfrak{x}_{1} \odot_{p} \mathfrak{x}_{2}$ of the vectors $\mathfrak{x}_{l}=\left(x_{1, l}, \ldots, x_{4, l}\right)^{T}, l=1,2$ is defined by the equation

$$
\begin{gathered}
\frac{\Gamma_{1}}{S_{1}\left(\mathfrak{x}_{1}, \mathfrak{x}_{2}\right)\left|\mathfrak{x}_{1}\right| p\left|\mathfrak{x}_{2}\right|_{p}} \mathfrak{x}_{1} \odot_{p} \mathfrak{x}_{2} \\
\left.\frac{S_{2}\left(\mathfrak{x}_{\left.1, \mathfrak{x}_{2}\right)} \xi_{1,1} \xi_{1,2}\left[\frac{x_{1,1}}{\xi_{1,1}}+\frac{x_{1,2}}{\xi_{1,2}}\right]\right.}{\Gamma_{2}}\left(\begin{array}{c}
x_{1,1} x_{1,2}-\xi_{1,1} \xi_{1,2} \\
x_{2,1} x_{2,2}-\xi_{2,1} \xi_{2,2} \\
\frac{\xi_{2,1} \xi_{2,2}}{\Gamma_{3}}\left[\frac{x_{2,1}}{\xi_{2,1}}+\frac{x_{2,2}}{\xi_{2,2}}\right]\left(\begin{array}{c}
x_{3,1} x_{3,2}-x_{4,1} x_{4,2} \\
x_{3,1} x_{4,2}+x_{4,1} x_{3,2}
\end{array}\right)
\end{array}\right)\right),
\end{gathered}
$$

otherwise (13) or (14) apply. 
We now move on to a more geometrical consideration. Let the $l_{4, p}$-spherical coordinate transformation $\mathrm{SPH}_{4, p}: \mathrm{M}_{4} \rightarrow \mathbb{R}^{4}$ be defined by

$$
\mathfrak{x}=\left(\begin{array}{l}
x_{1} \\
x_{2} \\
x_{3} \\
x_{4}
\end{array}\right)=S P H_{4, p}\left(r, \varphi_{1}, \varphi_{2}, \varphi_{3}\right)=r\left(\begin{array}{l}
\cos _{p}\left(\varphi_{1}\right) \\
\sin _{p}\left(\varphi_{1}\right) \cos _{p}\left(\varphi_{2}\right) \\
\sin _{p}\left(\varphi_{1}\right) \sin _{p}\left(\varphi_{2}\right) \cos _{p}\left(\varphi_{3}\right) \\
\sin _{p}\left(\varphi_{1}\right) \sin _{p}\left(\varphi_{2}\right) \sin _{p}\left(\varphi_{3}\right)
\end{array}\right)
$$

where, with $N_{p}(\varphi)=\left(|\cos \varphi|^{p}+|\sin \varphi|^{p}\right)^{1 / p}$, the $l_{p}$-trigonometric functions

$$
\cos _{p}(\varphi)=\cos \varphi / N_{p}(\varphi) \text { and } \sin _{p}(\varphi)=\sin \varphi / N_{p}(\varphi)
$$

are introduced in [16]. This transformation is a.e., invertible with the inverse transformation $S P H_{4, p}^{-1}\left(x_{1}, x_{2}, x_{3}, x_{4}\right)$ allowing the representations

$$
\begin{gathered}
r=\left(\left|x_{1}\right|^{p}+\left|x_{2}\right|^{p}+\left|x_{3}\right|^{p}+\left|x_{4}\right|^{p}\right)^{1 / p}=|\mathfrak{x}|_{p}, \\
\cos \varphi_{1}=\frac{N_{p}\left(\varphi_{1}\right) x_{1}}{r}, \sin \varphi_{1}=\frac{N_{p}\left(\varphi_{1}\right) \xi_{1}}{r}, \varphi_{1}=\arctan \frac{\xi_{1}}{x_{1}}, \\
\cos \varphi_{2}=\frac{N_{p}\left(\varphi_{2}\right) x_{2}}{\xi_{1}}, \sin \varphi_{2}=\frac{N_{p}\left(\varphi_{2}\right) x_{2}}{\xi_{1}}, \varphi_{2}=\arctan \frac{\xi_{2}}{x_{2}}, \\
\cos \varphi_{3}=\frac{N_{p}\left(\varphi_{3}\right) x_{3}}{\xi_{2}}, \sin \varphi_{3}=\frac{N_{p}\left(\varphi_{3}\right) x_{4}}{\xi_{2}}, \varphi_{3}=\arctan \frac{x_{4}}{x_{3}}
\end{gathered}
$$

where $|\mathfrak{x}|_{p}$ denotes the $l_{p}$-norm or, according to [19], the $l_{p}$-antinorm of $\mathfrak{x}$ if $p \geq 1$ or $0<p \leq 1$, respectively.

Definition 9. The spherical coordinate p-product of the vectors $\mathfrak{x}_{l}=S P H_{4, p}\left(r_{l}, \varphi_{1, l}, \varphi_{2, l}, \varphi_{3, l}\right)$, $l=1,2$ is defined as

$$
\begin{aligned}
S P H_{4, p}\left(r_{1}, \varphi_{1,1}, \varphi_{2,1}, \varphi_{3,1}\right) & *_{p} S P H_{4, p}\left(r_{2}, \varphi_{1,2}, \varphi_{2,2}, \varphi_{3,2}\right) \\
& =S P H_{4, p}\left(r_{1} r_{2}, \varphi_{1,1} \diamond \varphi_{1,2}, \varphi_{2,1} \diamond \varphi_{2,2}, \varphi_{3,1} \triangleright \varphi_{3,2}\right)
\end{aligned}
$$

where $\diamond$ and $\triangleright$ are defined as in (26) and (27), respectively.

Theorem 4. The spherical coordinate p-product of the vectors $\mathfrak{x}_{l}=S P H_{4, p}\left(r_{l}, \varphi_{1, l}, \varphi_{2, l}, \varphi_{3, l}\right)$, $l=1,2$ according to Definition 9 coincides with their geometric vector p-product according to Definition 8 .

Proof. We start from the representation

$$
\begin{aligned}
& S P H_{4, p}\left(r_{1} r_{2}, \varphi_{1,1} \diamond \varphi_{1,2}, \varphi_{2,1} \diamond \varphi_{2,2}, \varphi_{3,1} \triangleright \varphi_{3,2}\right) \\
&=r_{1} r_{2} \operatorname{diag}\left(\Theta_{1}, \Theta_{2}, \Theta_{3}, \Theta_{3}\right) \operatorname{Pol}_{(4)}\left(1, \varphi_{1,1} \diamond \varphi_{1,2}, \varphi_{2,1} \diamond \varphi_{2,2}, \varphi_{3,1} \triangleright \varphi_{3,2}\right)
\end{aligned}
$$

where

$$
\Theta_{1}=\frac{1}{N_{p}\left(\varphi_{1,1} \diamond \varphi_{1,2}\right)}, \Theta_{2}=\frac{\Theta_{1}}{N_{p}\left(\varphi_{2,1} \diamond \varphi_{2,2}\right)}, \Theta_{3}=\frac{\Theta_{2}}{N_{p}\left(\varphi_{3,1} \triangleright \varphi_{3,2}\right)} .
$$

Let $\varrho_{l}=\left\|\mathfrak{x}_{l}\right\|, l=1,2$ then

$$
\begin{aligned}
& S P H_{4, p}\left(r_{1} r_{2}, \varphi_{1,1} \diamond \varphi_{1,2}, \varphi_{2,1} \diamond \varphi_{2,2}, \varphi_{3,1} \triangleright \varphi_{3,2}\right) \\
& \quad=\frac{r_{1} r_{2}}{\varrho_{1} \varrho_{2}} \operatorname{diag}\left(\Theta_{1}, \Theta_{2}, \Theta_{3}, \Theta_{3}\right) \operatorname{Pol}_{(4)}\left(\varrho_{1} \varrho_{2}, \varphi_{1,1} \diamond \varphi_{1,2}, \varphi_{2,1} \diamond \varphi_{2,2}, \varphi_{3,1} \triangleright \varphi_{3,2}\right) .
\end{aligned}
$$

It follows from the properties of the trigonometric functions that

$$
N_{p}\left(\varphi_{l, 1} \diamond \varphi_{l, 2}\right)=N_{p}\left(\varphi_{l, 1}+\varphi_{l, 2}\right), l=1,2
$$


and

$$
N_{p}\left(\varphi_{3,1} \triangleright \varphi_{3,2}\right)=N_{p}\left(\varphi_{3,1}+\varphi_{3,2}\right) .
$$

Moreover,

$$
N_{p}\left(\varphi_{l, 1}+\varphi_{l, 2}\right)=\left(\left|s_{l, 1} c_{l, 2}+s_{l, 2} c_{l, 1}\right|^{p}+\left|c_{l, 1} c_{l, 2}-s_{l, 1} s_{l, 2}\right|^{p}\right)^{1 / p}
$$

where $s_{l, m}=\sin \varphi_{l, m}$ and $c_{l, m}=\cos \varphi_{l, m}, l=1,2,3, m=1,2$. Note that

$$
N_{p}\left(\varphi_{1,1}+\varphi_{1,2}\right)=\frac{N_{p}\left(\varphi_{1,1}\right) N_{p}\left(\varphi_{1,2}\right) \Gamma_{1}}{r_{1} r_{2}}
$$

and

$$
N_{p}\left(\varphi_{l, 1}+\varphi_{l, 2}\right)=\frac{N_{p}\left(\varphi_{l, 1}\right) N_{p}\left(\varphi_{l, 2}\right) \Gamma_{l}}{\xi_{l, 1} \xi_{l, 2}}, l=2,3 .
$$

Due to the representations

$$
x_{1, l}=r_{l} \cos p\left(\varphi_{1, l}\right) \text { and } x_{1, l}=\varrho_{l} \cos \varphi_{1, l}, l=1,2
$$

we have that

$$
N_{p}\left(\varphi_{1, l}\right)=r_{l} / \varrho_{l}, l=1,2
$$

Similarly,

$$
x_{2, l}=r_{l} \sin _{p}\left(\varphi_{1, l}\right) \cos _{p}\left(\varphi_{2, l}\right) \text { and } x_{2, l}=\varrho_{l} \sin \varphi_{1, l} \cos \varphi_{2, l}, l=1,2
$$

yields $N_{p}\left(\varphi_{1, l}\right) N_{p}\left(\varphi_{2, l}\right)=r_{l} / \varrho_{l}, l=1,2$, thus

$$
N_{p}\left(\varphi_{2, l}\right)=1, l=1,2 \text {. }
$$

Also, the representations

$$
x_{3, l}=r_{l} \sin _{p}\left(\varphi_{1, l}\right) \sin _{p}\left(\varphi_{2, l}\right) \cos _{p}\left(\varphi_{3, l}\right) \text { and } x_{3, l}=\varrho_{l} \sin \varphi_{1, l} \sin \varphi_{2, l} \cos \varphi_{3, l}
$$

result in $N_{p}\left(\varphi_{1, l}\right) N_{p}\left(\varphi_{2, l}\right) N_{p}\left(\varphi_{3, l}\right)=r_{l} / \varrho_{l}, l=1,2$, thus

$$
N_{p}\left(\varphi_{3, l}\right)=1, l=1,2 \text {. }
$$

So we get

$$
\Theta_{1}=\frac{\varrho_{1} \varrho_{2}}{\Gamma_{1}}, \Theta_{2}=\Theta_{1} \frac{\xi_{1,1} \xi_{1,2}}{\Gamma_{2}}, \Theta_{3}=\Theta_{2} \frac{\xi_{2,1} \xi_{2,2}}{\Gamma_{3}}
$$

and

$$
\begin{aligned}
& S P H_{4, p}\left(r_{1} r_{2}, \varphi_{1,1} \diamond \varphi_{1,2}, \varphi_{2,1} \diamond \varphi_{2,2}, \varphi_{3,1} \triangleright \varphi_{3,2}\right) \\
& =\left(\begin{array}{cccc}
\frac{\varrho_{1} \varrho_{2}}{\Gamma_{1}} & 0 & 0 & 0 \\
0 & \frac{\varrho_{1} \varrho_{2} \xi_{1,1} \tau_{1,2}}{\Gamma_{1} \Gamma_{2}} & 0 & 0 \\
0 & 0 & \frac{\varrho_{1} \varrho_{2} \xi_{1,1} \tau_{1,2} \xi_{2,1} \xi_{2,2}}{\Gamma_{1} \Gamma_{2} \Gamma_{3}} & 0 \\
0 & 0 & 0 & \frac{\varrho_{1} \varrho_{2} \xi_{1,1} \tau_{1,2} \tau_{2,1} \xi_{2,2}}{\Gamma_{1} \Gamma_{2} \Gamma_{3}}
\end{array}\right) \\
& \cdot \frac{r_{1} r_{2}}{\varrho_{1} \varrho_{2}} \operatorname{Pol}_{(4)}\left(\varrho_{1} \varrho_{2}, \varphi_{1,1} \diamond \varphi_{1,2}, \varphi_{2,1} \diamond \varphi_{2,2}, \varphi_{3,1} \triangleright \varphi_{3,2}\right) \\
& =\frac{r_{1} r_{2}}{\Gamma_{1}}\left(\begin{array}{cccc}
1 & 0 & 0 & 0 \\
0 & \frac{\xi_{1,1} \tau_{1,2}}{\Gamma_{2}} & 0 & 0 \\
0 & 0 & \frac{\xi_{1,1} \tau_{1,2} \xi_{2,1} \tau_{2,2}}{\Gamma_{2} \Gamma_{3}} & 0 \\
0 & 0 & 0 & \frac{\xi_{1,1} \tau_{1,2} \tau_{2,1} \tau_{2,2}}{\Gamma_{2} \Gamma_{3}}
\end{array}\right) \\
& \text { - } \operatorname{Pol}_{(4)}\left(\varrho_{1} \varrho_{2}, \varphi_{1,1} \diamond \varphi_{1,2}, \varphi_{2,1} \diamond \varphi_{2,2}, \varphi_{3,1} \triangleright \varphi_{3,2}\right)
\end{aligned}
$$


from where the result follows.

It is not hard now to check that the present number systems possess properties like (16). The results of this section can then be easily summarized as follows.

Proposition 2. For every $p$ greater than zero, the space $\left(\mathbb{R}^{4}, \boxplus, \bullet, \odot_{p}, \mathfrak{o}, \mathfrak{e}, \mathfrak{i}, \mathfrak{j}, \mathfrak{k}\right)$ is a realization of the four-complex algebraic structure $\mathbb{C}_{4}$.

\section{The Spherical Geometric Vector Product in Higher Dimensions}

According to the method developed in $[13,14]$ and continued in the previous sections, the key point for constructing multi-complex algebraic structures is the definition of a suitable geometric vector product. Such a definition is introduced and verified here.

For $m=1,2$, let $\mathfrak{x}_{m}=\left(x_{1, m}, \ldots, x_{n, m}\right)^{T} \in \mathbb{R}^{n}$ and

$$
\xi_{l, m}=\sqrt{x_{l+1, m}^{2}+\ldots+x_{n, m}^{2}}, l=0, \ldots, n-2, \xi_{n-1, m}=x_{n, m},
$$

and note that $\xi_{0, m}=r_{m}$ is the Euclidean norm of the vector $\mathfrak{x}_{m}$. Let further

$$
P_{l}\left(\mathfrak{x}_{1}, \mathfrak{x}_{2}\right)=\left(\frac{x_{1,1}}{\xi_{1,1}}+\frac{x_{1,2}}{\xi_{1,2}}\right) \cdots\left(\frac{x_{l-1,1}}{\xi_{l-1,1}}+\frac{x_{l-1,2}}{\xi_{l-1,2}}\right), l=2, \ldots, n-1
$$

and

$$
D_{l}\left(\mathfrak{x}_{1}, \mathfrak{x}_{2}\right)=x_{l, 1} x_{l, 2}-\xi_{l, 1} \xi_{l, 2}, l=1, \ldots, n-2
$$

Finally, we put

$$
S_{q}\left(\mathfrak{x}_{1}, \mathfrak{x}_{2}\right)=\prod_{l=1}^{q} S_{l}^{*}\left(\mathfrak{x}_{1}, \mathfrak{x}_{2}\right), q=1, \ldots, n-2
$$

where the sign $S_{l}^{*}\left(\mathfrak{x}_{1}, \mathfrak{x}_{2}\right)$ is defined as $S_{l}^{*}\left(\mathfrak{x}_{1}, \mathfrak{x}_{2}\right)=I_{l, 1}-I_{l, 2}$ with $I_{l, 1}=I_{[0, \pi]}\left(\varphi_{l, 1}+\varphi_{l, 2}\right)$, $I_{l, 2}=I_{(\pi, 2 \pi)}\left(\varphi_{l, 1}+\varphi_{l, 2}\right)$ and $\varphi_{l, m}=\arctan \frac{\tilde{l}_{l, m}}{x_{l, m}}, l=1, \ldots, n-1, m=1,2$.

Definition 10. Unless for

$$
\left(x_{n-1, m}, x_{n, m}\right)=(0,0), \ldots,\left(x_{2, m}, \ldots, x_{n, m}\right)=(0, \ldots, 0) \text { for at least one value of } m \in\{1,2\},
$$

the spherical geometric vector product of $\mathfrak{x}_{1}$ and $\mathfrak{x}_{2}$ is defined by

$$
\mathfrak{x}_{1} \odot \mathfrak{x}_{2}=\left(\begin{array}{l}
S_{1}\left(\mathfrak{x}_{1}, \mathfrak{x}_{2}\right) D_{1}\left(\mathfrak{x}_{1}, \mathfrak{x}_{2}\right) \\
S_{2}\left(\mathfrak{x}_{1}, \mathfrak{x}_{2}\right) P_{2}\left(\mathfrak{x}_{1}, \mathfrak{x}_{2}\right) D_{2}\left(\mathfrak{x}_{1}, \mathfrak{x}_{2}\right) \\
\vdots \\
S_{n-2}\left(\mathfrak{x}_{1}, \mathfrak{x}_{2}\right) P_{n-2}\left(\mathfrak{x}_{1}, \mathfrak{x}_{2}\right) D_{n-2}\left(\mathfrak{x}_{1}, \mathfrak{x}_{2}\right) \\
S_{n-2}\left(\mathfrak{x}_{1}, \mathfrak{x}_{2}\right) P_{n-1}\left(\mathfrak{x}_{1}, \mathfrak{x}_{2}\right)\left(x_{n-1,1} x_{n-1,2}-x_{n, 1} x_{n, 2}\right) \\
S_{n-2}\left(\mathfrak{x}_{1}, \mathfrak{x}_{2}\right) P_{n-1}\left(\mathfrak{x}_{1}, \mathfrak{x}_{2}\right)\left(x_{n, 1} x_{n-1,2}+x_{n-1,1} x_{n, 2}\right)
\end{array}\right) .
$$

Moreover, in particular, we put

$$
\left(x_{1}, \ldots, x_{n}\right)^{T} \odot(t, 0, \ldots, 0)^{T}=|t|\left(x_{1}, x_{2}, \ldots, x_{n}\right)^{T}, t \in \mathbb{R} .
$$

Let $M_{n}=[0, \infty) \times[0, \pi]^{\times(n-2)} \times[0,2 \pi)$ and define the usual $n$-dimensional polar coordinate transformation $\operatorname{Pol}_{(n)}: M_{n} \rightarrow \mathbb{R}^{n}$ by $x_{1}=r \cos \varphi_{1}, x_{2}=r \sin \varphi_{1} \cos \varphi_{2}, \ldots$, $x_{n-1}=r \sin \varphi_{1} \ldots \sin \varphi_{n-2} \cos \varphi_{n-1}$ and $x_{n}=r \sin \varphi_{1} \ldots \sin \varphi_{n-1}$. 
Definition 11. The spherical coordinate product of the vectors $\operatorname{Pol}_{(n)}\left(r_{m}, \varphi_{1, m}, \ldots, \varphi_{n-1, m}\right)$, $m=1,2$, is defined as

$$
\begin{aligned}
\operatorname{Pol}_{(n)}( & \left.r_{1}, \varphi_{1,1}, \ldots, \varphi_{n-1,1}\right) * \operatorname{Pol}_{(n)}\left(r_{2}, \varphi_{1,2}, \ldots, \varphi_{n-1,2}\right) \\
& =\operatorname{Pol}_{(n)}\left(r_{1} r_{2}, \varphi_{1,1} \diamond \varphi_{1,2}, \ldots, \varphi_{n-2,1} \diamond \varphi_{n-2,2}, \varphi_{n-1,1} \triangleright \varphi_{n-1,2}\right)
\end{aligned}
$$

where $\psi \diamond \vartheta$ and $\psi \triangleright \vartheta$ are introduced in (26) and (27), respectively.

Theorem 5. The spherical coordinate product of the vectors $\operatorname{Pol}_{(n)}\left(r_{m}, \varphi_{1, m}, \ldots, \varphi_{n-1, m}\right)=$ $\left(x_{1, m}, \ldots, x_{n, m}\right)^{T}=\mathfrak{x}_{m}, m=1,2$ according to Definition 11 coincides with their geometric vector product according to Definition 10.

Proof. According to Definition 11, the spherical coordinate product of the two vectors $\operatorname{Pol}_{(n)}\left(r_{1}, \varphi_{1,1}, \ldots, \varphi_{n-1,1}\right)$ and $\operatorname{Pol}_{(n)}\left(r_{2}, \varphi_{1,2}, \ldots, \varphi_{n-1,2}\right)$ allows the representation

$$
\operatorname{Pol}_{(n)}\left(r_{1} r_{2}, \varphi_{1,1} \diamond \varphi_{1,2}, \ldots, \varphi_{n-2,1} \diamond \varphi_{n-2,2}, \varphi_{n-1,1} \triangleright \varphi_{n-1,2}\right) /\left(r_{1} r_{2}\right)
$$

$$
=\left(\begin{array}{l}
\cos \left(\varphi_{1,1} \diamond \varphi_{1,2}\right) \\
\sin \left(\varphi_{1,1} \diamond \varphi_{1,2}\right) \cos \left(\varphi_{2,1} \diamond \varphi_{2,2}\right) \\
\sin \left(\varphi_{1,1} \diamond \varphi_{1,2}\right) \sin \left(\varphi_{2,1} \diamond \varphi_{2,2}\right) \cos \left(\varphi_{3,1} \diamond \varphi_{3,2}\right) \\
\vdots \\
\sin \left(\varphi_{1,1} \diamond \varphi_{1,2}\right) \ldots \sin \left(\varphi_{n-3,1} \diamond \varphi_{n-3,2}\right) \cos \left(\varphi_{n-2,1} \diamond \varphi_{n-2,2}\right) \\
\sin \left(\varphi_{1,1} \diamond \varphi_{1,2}\right) \ldots \sin \left(\varphi_{n-3,1} \diamond \varphi_{n-3,2}\right) \sin \left(\varphi_{n-2,1} \diamond \varphi_{n-2,2}\right) \cos \left(\varphi_{n-1,1} \triangleright \varphi_{n-1,2}\right) \\
\sin \left(\varphi_{1,1} \diamond \varphi_{1,2}\right) \ldots \sin \left(\varphi_{n-3,1} \diamond \varphi_{n-3,2}\right) \sin \left(\varphi_{n-2,1} \diamond \varphi_{n-2,2}\right) \sin \left(\varphi_{n-1,1} \triangleright \varphi_{n-1,2}\right)
\end{array}\right) .
$$

On using the notation $c_{l, m}=\cos \varphi_{l, m}$ and $s_{l, m}=\sin \varphi_{l, m}, m=1,2$ we have

$$
\begin{aligned}
\cos \left(\varphi_{l, 1} \diamond \varphi_{l, 2}\right) & =\left(c_{l, 1} c_{l, 2}-s_{l, 1} s_{l, 2}\right)\left[I_{l, 1}-I_{l, 1}\right], l=1, \ldots, n-2 \\
\cos \left(\varphi_{n-1,1} \triangleright \varphi_{n-1,2}\right) & =c_{n-1,1} c_{n-1,2}-s_{n-1,1} s_{n-1,2}
\end{aligned}
$$

and

$$
\begin{aligned}
\sin \left(\varphi_{l, 1} \diamond \varphi_{l, 2}\right) & =\left(s_{l, 1} c_{l, 2}+c_{l, 1} s_{l, 2}\right)\left[I_{l, 1}-I_{l, 2}\right], l=1, \ldots, n-2 \\
\sin \left(\varphi_{n-1,1} \triangleright \varphi_{n-1,2}\right) & =s_{n-1,1} c_{n-1,2}+c_{n-1,1} s_{n-1,2} .
\end{aligned}
$$

With $S_{l}=S_{l}\left(\mathfrak{x}_{1}, \mathfrak{x}_{2}\right), l=1, \ldots, n-2$, it follows that

$$
\begin{aligned}
& \operatorname{Pol}_{(n)}\left(r_{1} r_{2}, \varphi_{1,1} \diamond \varphi_{1,2}, \ldots, \varphi_{n-2,1} \diamond \varphi_{n-2,2}, \varphi_{n-1,1} \triangleright \varphi_{n-1,2}\right) /\left(r_{1} r_{2}\right) \\
& =\left(\begin{array}{l}
S_{1}\left(c_{1,1} c_{1,2}-s_{1,1} s_{1,2}\right) \\
S_{2}\left(s_{1,1} c_{1,2}+c_{1,1} s_{1,2}\right)\left(c_{2,1} c_{2,2}-s_{2,1} s_{2,2}\right) \\
S_{3}\left(s_{1,1} c_{1,2}+c_{1,1} s_{1,2}\right)\left(s_{2,1} c_{2,2}+c_{2,1} s_{2,2}\right)\left(c_{3,1} c_{3,2}-s_{3,1} s_{3,2}\right) \\
\vdots \\
S_{n-2}\left(s_{1,1} c_{1,2}+c_{1,1} s_{1,2}\right) \ldots\left(s_{n-3,1} c_{n-3,2}+c_{n-3,1} s_{n-3,2}\right)\left(c_{n-2,1} c_{n-2,2}-s_{n-2,1} s_{n-2,2}\right) \\
S_{n-2}\left(s_{1,1} c_{1,2}+c_{1,1} s_{1,2}\right) \ldots\left(s_{n-2,1} c_{n-2,2}+c_{n-2,1} s_{n-2,2}\right)\left(c_{n-1,1} c_{n-1,2}-s_{n-1,1} s_{n-1,2}\right) \\
S_{n-2}\left(s_{1,1} c_{1,2}+c_{1,1} s_{1,2}\right) \ldots\left(s_{n-2,1} c_{n-2,2}+c_{n-2,1} s_{n-2,2}\right)\left(s_{n-1,1} c_{n-1,2}+c_{n-1,1} s_{n-1,2}\right)
\end{array}\right) .
\end{aligned}
$$

By inverse $n$-dimensional polar coordinate transformation,

$$
c_{1, m}=\frac{x_{1, m}}{\xi_{1-1, m}}, s_{1, m}=\frac{\xi_{1, m}}{\xi_{1-1, m}}, m=1,2, l=1, \ldots, n-1 .
$$

Thus, 


$$
\begin{aligned}
\operatorname{Pol}_{(n)}\left(r_{1} r_{2}, \varphi_{1,1} \diamond \varphi_{1,2}, \ldots, \varphi_{n-2,1} \diamond \varphi_{n-2,2}, \varphi_{n-1,1} \triangleright \varphi_{n-1,2}\right) \\
=r_{1} r_{2}\left(\begin{array}{l}
S_{1} \frac{x_{1,1} x_{1,2}-\xi_{1,1} \xi_{1,2}}{r_{1} r_{2}} \\
S_{2} \frac{\xi_{1,1} x_{1,2}+x_{1,1} \xi_{1,2}}{r_{1} r_{2}} \frac{x_{2,1} x_{2,2}-\xi_{2,1} \xi_{2,2}}{\xi_{1,1} \xi_{1,2}} \\
S_{3} \frac{\xi_{1,1} x_{1,2}+x_{1,1} \xi_{1,2}}{r_{1} r_{2}} \frac{\xi_{2,1} x_{2,2}+x_{2,1} \xi_{2,2}}{\xi_{1,1} \xi_{1,2}} \frac{x_{3,1} x_{3,2}-\xi_{3,1} \xi_{3,2}}{\xi_{2,1} \xi_{2,2}} \\
\vdots \\
S_{n-2} \frac{\xi_{1,1} x_{1,2}+x_{1,1} \xi_{1,2}}{r_{1} r_{2}} \cdots \frac{\xi_{n-3,1} x_{n-3,2}+x_{n-3,1} \xi_{n-3,2}}{\xi_{n-4,1} \xi_{n-4,2}} \frac{x_{n-2,1} x_{n-2,2}-\xi_{n-2,1} \xi_{n-2,2}}{\xi_{n-3,1} \xi_{n-3,2}} \\
S_{n-2} \frac{\xi_{1,1} x_{1,2}+x_{1,1} \xi_{1,2}}{r_{1} r_{2}} \cdots \frac{\xi_{n-2,1} x_{n-2,2}+x_{n-2,1} \xi_{n-2,2}}{\xi_{n-3,1} \xi_{n-3,2}} \frac{x_{n-1,1} x_{n-1,2}-\xi_{n-1,1} \xi_{n-1,2}}{\xi_{n-2,1} \xi_{n-2,2}} \\
S_{n-2} \frac{\xi_{1,1} x_{1,2}+x_{1,1} \xi_{1,2}}{r_{1} r_{2}} \cdots \frac{\xi_{n-2,1} x_{n-2,2}+x_{n-2,1} \xi_{n-2,2}}{\xi_{n-3,1} \xi_{n-3,2}} \frac{\xi_{n-1,1} x_{n-1,2}+x_{n-1,1} \xi_{n-1,2}}{\xi_{n-2,1} \xi_{n-2,2}}
\end{array}\right) \\
=\left(\begin{array}{l}
S_{1}\left(x_{1,1} x_{1,2}-\xi_{1,1} \xi_{1,2}\right) \\
S_{2}\left(\frac{x_{1,2}}{\xi_{1,2}}+\frac{x_{1,1}}{\xi_{1,1}}\right)\left(x_{2,1} x_{2,2}-\xi_{2,1} \xi_{2,2}\right) \\
S_{3}\left(\frac{x_{1,2}}{\xi_{1,2}}+\frac{x_{1,1}}{\xi_{1,1}}\right)\left(\frac{x_{2,2}}{\xi_{2,2}}+\frac{x_{2,1}}{\xi_{2,1}}\right)\left(x_{3,1} x_{3,2}-\xi_{3,1} \xi_{3,2}\right) \\
\vdots \\
S_{n-2}\left(\frac{x_{1,2}}{\xi_{1,2}}+\frac{x_{1,1}}{\xi_{1,1}}\right) \cdots\left(\frac{x_{n-3,2}}{\xi_{n-3,2}}+\frac{x_{n-3,1}}{\xi_{n-3,1}}\right)\left(x_{n-2,1} x_{n-2,2}-\xi_{n-2,1} \xi_{n-2,2}\right) \\
S_{n-2}\left(\frac{x_{1,2}}{\xi_{1,2}}+\frac{x_{1,1}}{\xi_{1,1}}\right) \cdots\left(\frac{x_{n-2,2}}{\xi_{n-2,2}}+\frac{x_{n-2,1}}{\xi_{n-2,1}}\right)\left(x_{n-1,1} x_{n-1,2}-\xi_{n-1,1} \xi_{n-1,2}\right) \\
S_{n-2}\left(\frac{x_{1,2}}{\xi_{1,2}}+\frac{x_{1,1}}{\xi_{1,1}}\right) \cdots\left(\frac{x_{n-2,2}}{\xi_{n-2,2}}+\frac{x_{n-2,1}}{\xi_{n-2,1}}\right)\left(\xi_{n-1,1} x_{n-1,2}+x_{n-1,1} \xi_{n-1,2}\right)
\end{array}\right)
\end{aligned}
$$

from where the result follows.

\section{Multi-Complex Numbers}

Having defined the geometric vector product in higher dimensions, we are now in a position to introduce the notion of a multi-complex structure. Thereby, we restrict our consideration to the particular case where the basic vector space is just the Euclidean vector space $\mathbb{R}^{n}$. To this end, the definitions of the geometric vector power and its complex exponential function can be taken over from Definition 2 word for word for the higher dimensional case. Let $\mathfrak{e}=\left(1,0_{n-1}^{T}\right)^{T}, i_{l}=\left(0_{l}^{T}, 1,0_{n-l-1}^{T}\right)^{T}, l=1, \ldots, n-2, i_{n-1}=\left(0_{n-1}^{T}, 1\right)^{T}$.

Example 6. Particular multiplication results are

$$
i_{l} \odot i_{m}=-\mathfrak{e}, l \neq m \text {. }
$$

Example 7. If $\mathfrak{x}=x_{2} \mathfrak{i}_{1}+\ldots+x_{n} \mathfrak{i}_{n-1}$ and $\mathfrak{\xi}=\sqrt{x_{2}^{2}+\ldots+x_{n}^{2}}$ then

$$
\mathfrak{x}^{2 q}=(-1)^{q} \mathfrak{\xi}^{2 q} \mathfrak{e}, \quad \mathfrak{x}^{2 q+1}=(-1)^{q} \mathfrak{\xi}^{2 q} \mathfrak{x}, q=0,1,2, \ldots
$$

Example 8. The following Euler type formulas are true:

$$
e_{\odot}^{\tau \mathfrak{h}}=(\cos \tau) \mathfrak{e}+(\sin \tau) \mathfrak{h}, \tau \in \mathbb{R}, \mathfrak{h} \in\left\{i_{1}, \ldots, i_{n-1}\right\}
$$

and

$$
e_{\odot}^{\mathfrak{x}}=(\cos \xi) \mathfrak{e}+(\sin \xi) \frac{\mathfrak{x}}{\mathfrak{\xi}}
$$

where $\mathfrak{x}$ and $\xi$ are as in Example 7.

Definition 12. Let $\mathbb{R}^{n}$ be the n-dimensional Euclidean vector space endowed with the common vector addition $\boxplus$ and the geometric vector product $\odot$ according to Definition 10 . Then $\mathbb{C}_{n}=$ $\left(\mathbb{R}^{n}, \oplus, \boxplus, 0_{n}, \mathfrak{e}_{n}, i_{1}, \ldots, i_{n-1}\right)$ is called the space of $n$-complex numbers, $0_{n}$ and $\mathfrak{e}_{n}$ are accordingly the neutral elements of addition and multiplication, and $i_{1}, \ldots, i_{n-1}$ are called the imaginary units of this space. 


\section{Discussion}

In the previous section, the definition of a four-complex algebraic structure given in Section 2 was extended to higher dimensions. In all of these cases, the questions of the existence and uniqueness of realizations of such algebraic structures arise immediately. For dimensions two and three, these questions were answered in $[13,14]$. In the case of dimension two and $p=2$, some authors do not explicitly state that the multiplication is linked to a (5) analog calculation rule. However, to state this explicitly leads in a certain way to the introduction of the vector product (7), which was generalized for dimensions three and four in Formulas (8) and (12), respectively. A multivariate generalization of this geometric vector product is given in Section 7 and serves as the key instrument for the construction of multi-complex structures. It is indicated in the text close to Formula (6) and in Remark 3 that, consequently, some of the usual algebraic rules known from usual complex numbers are replaced with new ones, here. This is the reason why the classification of real division algebras according to [18] does not apply, here.

Funding: The APC was funded by Deutsche Forschungsgemeinschaft and Universität Rostock within the funding programme Open Access Publishing.

Acknowledgments: The author is grateful to the Reviewers for their valuable comments and suggestions.

Conflicts of Interest: The author declares no conflict of interest.

\section{Appendix A}

As noted in [14,15], numerous theoretically and practically significant results in connection with complex numbers have been derived on a partly non-rigorous or 'alchemical' basis over a historically long period of time. An often even today met in the literature lack of rigor in the presentation of (usual) complex numbers was closed in [13] by a completely formally correct introduction of these numbers as a particular realization of a general algebraic structure. The consequences of this for the formally completely correct representation of some applications of complex numbers will be discussed in this section.

Different possibilities to represent systems of mathematical objects that are called complex numbers were derived during the centuries. A system of complex numbers will be considered here to be an algebraic structure $\mathbb{C}_{2}=(\mathbb{C}, \oplus, \odot, \mathfrak{o}, \mathfrak{e}, \mathfrak{i})$ where $\mathbb{C}$ is a non-empty set and $\oplus, \odot$ are commutative and associative binary operations acting from $\mathbb{C} \times \mathbb{C}$ to $\mathbb{C}$ in a way such that $(\mathbb{C}, \oplus)$ and $(\mathbb{C}, \odot)$ are Abelian groups with neutral elements $\mathfrak{o}$ and $\mathfrak{e}$, respectively, and the two elements $\mathfrak{e}$ and $\mathfrak{i}$ from $\mathbb{C}$ satisfy the equation $\mathfrak{i} \odot \mathfrak{i}=-\mathfrak{e}$.

For simplicity, we restrict our consideration throughout this section to the most classical case where

$$
\begin{gathered}
\mathbb{C}=\left\{\left(\begin{array}{l}
x \\
y
\end{array}\right), x \in \mathbb{R}, y \in \mathbb{R}\right\},\left(\begin{array}{l}
x \\
y
\end{array}\right) \oplus\left(\begin{array}{l}
x^{\prime} \\
y^{\prime}
\end{array}\right)=\left(\begin{array}{l}
x+x^{\prime} \\
y+y^{\prime}
\end{array}\right), \\
\left(\begin{array}{l}
x \\
y
\end{array}\right) \odot\left(\begin{array}{l}
x^{\prime} \\
y^{\prime}
\end{array}\right)=\left(\begin{array}{l}
x x^{\prime}-y y^{\prime} \\
x y^{\prime}+x^{\prime} y
\end{array}\right)
\end{gathered}
$$

and where + and - mean addition and subtraction of real numbers. For other realizations of $\mathbb{C}_{2}$ and for its generalizations $\mathbb{C}_{p}, p>0$, see [13].

\section{Appendix A.1. Solutions to Quadratic Equations}

The equation $x^{2}+p x+q=0, x \in \mathbb{R}$ where $p$ and $q$ are real constants has no solution for $x$ if $q-p^{2} / 4>0$. For this case, let us consider the equation

$$
\left(\begin{array}{l}
x \\
y
\end{array}\right) \odot\left(\begin{array}{l}
x \\
y
\end{array}\right)+p\left(\begin{array}{l}
x \\
y
\end{array}\right)+q \mathfrak{e}=\mathfrak{o},\left(\begin{array}{l}
x \\
y
\end{array}\right) \in \mathbb{R}^{2} .
$$


In other words, we consider the equation system

$$
\begin{aligned}
x^{2}-y^{2}+p x+q & =0, \\
2 x y+p y & =0
\end{aligned}
$$

which, for $y \neq 0$, has the solutions

$$
\left(\begin{array}{l}
x \\
y
\end{array}\right) \in\left\{\left(\begin{array}{c}
-p / 2 \\
\sqrt{-p^{2} / 4+q}
\end{array}\right),\left(\begin{array}{c}
-p / 2 \\
-\sqrt{-p^{2} / 4+q}
\end{array}\right)\right\} .
$$

The traditional way to write this result is to call $x_{1}=-p / 2+i \sqrt{-p^{2} / 4+q}$ as well as $x_{2}=-p / 2-i \sqrt{-p^{2} / 4+q}$ the solutions of the first equation in this section. Here, however, the quantity $i=\sqrt{-1}$ is mathematically not defined, which, if one does not consider the historical development, is actually an absurdity for an exact science. The use of the usual way of dealing with $i$, clearly, remains unaffected by this remark. The key point of the present approach is its consequent start from a vector space consideration in combination with the introduction of the product $\odot$. For a more general approach to quadratic equations, we refer to [15].

\section{Appendix A.2. Derivatives of Complex Valued Functions}

Let $D$ be an open subset of $\mathbb{C}$. We call a function $f: D \rightarrow \mathbb{C}$ differentiable in $z_{0} \in D$ if the ratio $\left(f(z)-f\left(z_{0}\right)\right) \oslash\left(z-z_{0}\right)$ approaches a limit as $z$ approaches $z_{0}$. In this case, it is natural to write

$$
f^{\prime}\left(z_{0}\right)=\lim _{z \rightarrow z_{0}}\left(f(z)-f\left(z_{0}\right)\right) \oslash\left(z-z_{0}\right) .
$$

Hereby, the ratio of two complex numbers $z_{l}=\left(x_{l}, y_{l}\right)^{T}, l=1,2$ is defined according to [13] as

$$
z_{1} \oslash z_{2}=\frac{\left\|z_{1}\right\|}{\left\|z_{2}\right\|} \frac{\left(\begin{array}{l}
x_{1} x_{2}+y_{1} y_{2} \\
x_{2} y_{1}-y_{2} x_{1}
\end{array}\right)}{\left\|\left(\begin{array}{l}
x_{1} x_{2}+y_{1} y_{2} \\
x_{2} y_{1}-y_{2} x_{1}
\end{array}\right)\right\|}
$$

with $\|z\|$ meaning the Euclidean norm of the vector $z \in \mathbb{R}^{2}$. We remark that

$$
\begin{aligned}
z \oslash\left(\begin{array}{l}
1 \\
0
\end{array}\right) & =z, \\
\left(\begin{array}{l}
x \\
y
\end{array}\right) \oslash\left(\begin{array}{l}
0 \\
1
\end{array}\right) & =\left(\begin{array}{c}
y \\
-x
\end{array}\right)=\left.\left(\begin{array}{cc}
\cos \alpha & \sin \alpha \\
-\sin \alpha & \cos \alpha
\end{array}\right)\left(\begin{array}{l}
x \\
y
\end{array}\right)\right|_{\alpha=\pi / 2} .
\end{aligned}
$$

While the first of these two divisions describes the identical map, the second one means a clockwise rotation through $\pi / 2$.

The function $f$ will be called holomorphic in $D$ if it is differentiable in every $z_{0}$ from $D$. If such function is given by

$$
f\left(\left(\begin{array}{l}
x \\
y
\end{array}\right)\right)=\left(\begin{array}{l}
u(x, y) \\
v(x, y)
\end{array}\right),\left(\begin{array}{l}
x \\
y
\end{array}\right) \in D
$$

then it follows that the well known Cauchy-Riemann differential equations hold. To prove this, we consider points $z_{l}=z_{0}+z_{l, h}, l=1,2$ where

$$
z_{1, h}=\left(\begin{array}{l}
h \\
0
\end{array}\right) \text { and } z_{2, h}=\left(\begin{array}{l}
0 \\
h
\end{array}\right)
$$


with values of $h$ tending to zero, and denote partial derivatives with respect to $x$ or $y$ of a function $w(x, y)$ by $w_{x}$ and $w_{y}$, respectively. Then

$$
\begin{aligned}
\left(f\left(z_{1}\right)-f\left(z_{0}\right)\right) \oslash\left(z_{1}-z_{0}\right) & =\left(\begin{array}{c}
u\left(x_{0}+h, y_{0}\right)-u\left(x_{0}, y_{0}\right) \\
v\left(x_{0}+h, y_{0}\right)-v\left(x_{0}, y_{0}\right)
\end{array}\right) \oslash z_{1, h} \\
& =\left(\begin{array}{l}
u_{x}\left(x_{0}+\delta h, y_{0}\right) \\
v_{x}\left(x_{0}+\delta h, y_{0}\right)
\end{array}\right) \oslash \mathfrak{e} \rightarrow\left(\begin{array}{l}
u_{x}\left(x_{0}, y_{0}\right) \\
v_{x}\left(x_{0}, y_{0}\right)
\end{array}\right), h \rightarrow 0
\end{aligned}
$$

where $\delta$ is a certain number from the interval $(0,1)$. Similarly,

$$
\begin{aligned}
\left(f\left(z_{2}\right)-f\left(z_{0}\right)\right) \oslash\left(z_{2}-z_{0}\right) & =\left(\begin{array}{c}
u\left(x_{0}+h, y_{0}\right)-u\left(x_{0}, y_{0}\right) \\
v\left(x_{0}+h, y_{0}\right)-v\left(x_{0}, y_{0}\right)
\end{array}\right) \oslash z_{2, h} \\
& =\left(\begin{array}{c}
u_{y}\left(x_{0}+\delta h, y_{0}\right) \\
v_{y}\left(x_{0}+\delta h, y_{0}\right)
\end{array}\right) \oslash(0,1)^{T} \rightarrow\left(\begin{array}{c}
v_{y}\left(x_{0}, y_{0}\right) \\
-u_{y}\left(x_{0}, y_{0}\right)
\end{array}\right), h \rightarrow 0 .
\end{aligned}
$$

Appendix A.3. Avoiding a Computational Conflict

The basis for the complex algebraic structure $\mathbb{C}_{2}$ property

$$
\mathfrak{i} \odot \mathfrak{i}=-\mathfrak{e}
$$

is commonly written in usual (mathematically alchemical) terms of complex numbers as

$$
\sqrt{-1} \cdot \sqrt{-1}=-1
$$

Using notation $\sqrt{-1}=(-1)^{1 / 2}$ and hoping or assuming (in a certain mathematically non-rigorous sense) that calculation rules from the real number system can be used for the non-defined quantity $\sqrt{-1}$ leads to the well known two contradictory results

$$
(-1)^{1 / 2} \cdot(-1)^{1 / 2}=(-1)^{1 / 2+1 / 2}=-1 \text { and }(-1)^{1 / 2} \cdot(-1)^{1 / 2}=((-1) \cdot(-1))^{1 / 2}=1 .
$$

Occasionally, authors comment on this conflict with a completely unusual call for mathematics to be vigilant, but without giving an in-depth explanation. Acting within $\mathbb{C}_{2}$, however, does not provoke such a conflict.

Appendix A.4. Factorization of Characteristic Functions of Sums of Independent Random Variables

The characteristic function of a random variable $\mathfrak{x}$ is defined as

$$
\varphi_{\mathfrak{x}}(t)=\mathbb{E} e^{i t \mathfrak{x}}=\left(\begin{array}{c}
\mathbb{E} \cos (t \mathfrak{x}) \\
\mathbb{E} \sin (t \mathfrak{x})
\end{array}\right), t \in \mathbb{R} .
$$

Let $\mathfrak{x}_{1}$ and $\mathfrak{x}_{2}$ be independent random variables defined on a joint probability space, then

$$
\begin{aligned}
\varphi_{\mathfrak{x}_{1}+\mathfrak{x}_{2}}(t) & =\mathbb{E}\left(\begin{array}{c}
\cos \left(t \mathfrak{x}_{1}\right) \cos \left(t \mathfrak{x}_{2}\right)-\sin \left(t \mathfrak{x}_{1}\right) \sin \left(t \mathfrak{x}_{2}\right) \\
\cos \left(t \mathfrak{x}_{1}\right) \sin \left(t \mathfrak{x}_{2}\right)+\sin \left(t \mathfrak{x}_{1}\right) \cos \left(t \mathfrak{x}_{2}\right)
\end{array}\right) \\
& =\mathbb{E}\left(\begin{array}{c}
\cos \left(t \mathfrak{x}_{1}\right) \\
\sin \left(t \mathfrak{x}_{1}\right)
\end{array}\right) \odot\left(\begin{array}{c}
\cos \left(t \mathfrak{x}_{2}\right) \\
\sin \left(t \mathfrak{x}_{2}\right)
\end{array}\right) \\
& =\int\left[\int e^{i t z_{1}} \odot e^{i t z_{2}} P_{\mathfrak{x}_{2}}\left(d x_{2}\right)\right] P_{\mathfrak{x}_{1}}\left(d x_{1}\right) \\
& =\varphi_{\mathfrak{x}_{1}}(t) \odot \varphi_{\mathfrak{x}_{2}}(t)=\varphi_{\mathfrak{x}_{1}}(t) \varphi_{\mathfrak{x}_{2}}(t)
\end{aligned}
$$

reproving a well-known property of characteristic functions based upon the completely correct mathematical definition of the notion of complex numbers given in [13]. 


\section{Appendix A.5. Why Imaginary Numbers Are no More Imaginary}

At the hand of some examples, we have seen in the previous sections that several basic mathematical statements that commonly make use of the imaginary unit $i=\sqrt{-1}$ can be reformulated in a verifiable mathematically fully formally correct manner without making use of $i$. By studying a particular realization of an abstract algebraic complex structure in combination with a product suitably defined on it, the imaginary (or 'mathematically alchemical') character of the imaginary unit has disappeared. To conclude this section, with certain equal rights, one could say that negative numbers are not 'negative' in a corresponding sense.

\section{References}

1. Conway, J.H.; Smith, D. On Quaternions and Octonions: Their Geometry, Arithmetic and Symmetry; A K Peters/CRC Press: Boca Raton, FL, USA, 2003.

2. Kuipers, J.B. Quaternions and Rotation Sequences; Princeton University Press: Princeton, NJ, USA, 2002.

3. Olariu, S. Complex Numbers in n Dimensions; Elsevier: Amsterdam, The Netherlands; Boston, MA, USA; Tokyo, Japan, 2002.

4. Fjelstad, P.; Gal, S.G. N-dimensional hyperbolic complex numbers. Adv. Appl. Clifford Algebr. 1998, 8, 47-68. [CrossRef]

5. Muralidhar, K. Algebra of complex vectors and applications in electromagnetic theory and quantum mechanics. Mathematics 2015, 3, 781-842. [CrossRef]

6. Price, G.B. An Introduction to Multicomplex Spaces and Functions; Marcel Dekker: New York, NY, USA, 1991.

7. Lavoie, R.G.; Marchildon, L.; Rochon, D. Infinite-Dimensional Bicomplex Hilbert Spaces. Ann. Funct. Anal. 2010, 1, 75-91. [CrossRef]

8. Lavoie, R.G.; Marchildon, L.; Rochon, D. Finite-Dimensional Bicomplex Hilbert Spaces. Adv. Appl. Clifford Algebr. 2011, 21, 561-581. [CrossRef]

9. Adler, S.L. Quaternionic Quantum Mechanics and Quantum Fields; Oxford University Press: Oxford, UK, 1995.

10. Rochon, C.; Tremblay, S. Bicomplex Quantum Mechanics, I. The generalized Schrödinger equation. Adv. Appl. Clifford Algebr. 2004, 14, 231-248. [CrossRef]

11. Rochon, C.; Tremblay, S. Bicomplex Quantum Mechanics, II. The Hilbert Space. Adv. Appl. Clifford Algebr. 2006, 16, 135-157. [CrossRef]

12. Mursaleen, M.; Nasiruzzaman, M.; Srivastava, H.M. Approximation by bicomplex beta operators in compact BC-disks. Math Methods Appl. Sci. 2016, 39, 2916-2929. [CrossRef]

13. Richter, W.-D. On $l_{p}$-complex numbers. Symmetry 2020, 12, 877. [CrossRef]

14. Richter, W.-D. Three-complex numbers and related algebraic structures. Symmetry 2021, 13, 342. [CrossRef]

15. Richter, W.-D. Complex numbers related to semi-antinorms, ellipses or matrix homogeneous functionals. Axioms 2021, 10, 340. [CrossRef]

16. Richter, W.-D. Generalized spherical and simplicial coordinates. J. Math. Anal. Appl. 2007, 336, 1187-1202. [CrossRef]

17. Neutsch, W. Coordinates; DeGruyter: Berlin, Germany, 1996.

18. Palais, R.S. The Classification of Real Division Algebras. Am. Math. Mon. 1968, 75, 366-368. [CrossRef]

19. Moszyńska, M.; Richter, W.-D. Reverse triangle inequality. Antinorms and semi-antinorms. Stud. Sci. Math. Hung. 2012, 49, 120-138. [CrossRef] 